UNIVERSITÉ DU QUÉBEC À CHICOUTIMI

\author{
MÉMOIRE \\ PRÉSENTÉ \\ COMME EXIGENCE PARTIELLE \\ DE LA MAITTRISE EN ÉDUCATION
}

\author{
PAR \\ MARIE-CLAUDE BOUCHARD \\ BACHELIÈRE EN SCIENCES INFIRMIÈRES
}

Les infirmières : demandes sociales et enjeux de formation 


\section{Bibliothèque}

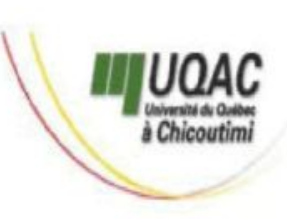

\section{Mise en garde/Advice}

Afin de rendre accessible au plus Motivated by a desire to make the grand nombre le résultat des results of its graduate students' travaux de recherche menés par ses research accessible to all, and in étudiants gradués et dans l'esprit des accordance with the rules règles qui régissent le dépôt et la governing the acceptation and diffusion des mémoires et thèses diffusion of dissertations and produits dans cette Institution, theses in this Institution, the I'Université du Québec à Université du Québec à Chicoutimi (UQAC) est fière de Chicoutimi (UQAC) is proud to rendre accessible une version make a complete version of this complète et gratuite de cette œuvre. work available at no cost to the reader.

L'auteur conserve néanmoins la The author retains ownership of the propriété du droit d'auteur qui copyright of this dissertation or protège ce mémoire ou cette thèse. thesis. Neither the dissertation or Ni le mémoire ou la thèse ni des thesis, nor substantial extracts from extraits substantiels de ceux-ci ne it, may be printed or otherwise peuvent être imprimés ou autrement reproduced without the author's reproduits sans son autorisation. permission. 


\section{RÉSUMÉ}

La formation infirmière est encore aujourd'hui au centre de nombreuses controverses. Au début des années 90 , ce sont deux réformes qui relancent le débat sur la formation infirmière, soient la réforme de la Santé et des Services sociaux, et la réforme de l'Éducation. Plusieurs changements sont à prévoir.

Dans le domaine de la santé, la nouvelle philosophie adoptée par le ministère de la Santé et des Services sociaux, davantage axée sur la prévention et la promotion, apporte des transformations majeures au réseau. Plusieurs organismes ont analysé les nouveaux besoins en soins de santé et ont pris position quant au niveau de formation de base en soins infirmiers permettant de répondre à ces demandes. Les nouveaux modes de distribution de soins ainsi que les besoins croissants de la population nécessitent une pratique infirmière plus autonome et requièrent des compétences multiples. L'infirmière doit avoir une formation scientifique, une plus grande autonomie, un sens des responsabilités plus développé, ainsi que des connaissances en enseignement, en animation, en gestion et en coordination. Grâce à une analyse de contenu effectuée à partir des discours que les organismes ont publiés, nous avons pu mettre en relief les besoins en soins de santé de la population et les compétences qu'ils jugeaient essentielles pour y répondre.

Ces nouvelles orientations adoptées par le ministère de la Santé et des Services sociaux du Québec, au début des années 90, apportent d'importantes modifications au réseau de la santé. Ces dernières ont un impact considérable sur la pratique infirmière actuelle et future puisqu'elles exigent l'élargissement du champ de compétences professionnelles.

Or, en éducation, le Ministère décide d'adopter une nouvelle philosophie et de revoir les programmes d'enseignement selon l'approche par compétences. C'est donc suite aux analyses de situation de travail que débute l'élaboration du programme d'enseignement collégial en soins infirmiers selon l'approche par compétences. Par cette approche, le ministère de l'Éducation espère répondre plus adéquatement aux besoins de maind'oeuvre des employeurs. Cependant, au Québec, il existe deux niveaux de formation, l'un collégial et l'autre universitaire. Dans le cadre de cette recherche, la question qui se 
pose est de savoir si la formation collégiale en soins infirmiers peut répondre aux nouvelles demandes sociales.

Une analyse de contenu, à partir de documents officiels publiés par des organismes qui s'intéressent à la formation infirmière, nous permet d'apporter quelques éléments de réponse. Par ailleurs, l'analyse du nouveau programme au collégial en soins infirmiers selon l'approche par compétences, nous permet de voir dans quelle mesure le ministère de l'Éducation a tenu compte des nouvelles orientations sociales lors de l'élaboration de ce nouveau programme.

Les observations portant sur ces deux niveaux d'analyse ont été révélatrices. En effet, les résultats obtenus nous ont permis, en plus de mettre en parallèle les nouveaux besoins en soins de santé et les compétences visées par le nouveau programme de formation collégiale, de mettre en évidence les limites de la formation collégiale en soins infirmiers par rapport à ces nouvelles demandes. En effet, l'infirmière qui désire exercer dans le secteur communautaire devra poursuivre sa formation à l'université. Un continuum de formation sur cinq ans, (diplôme d'études collégiales sur trois ans et baccalauréat universitaire en deux ans), a été proposé par le ministère de l'Éducation en janvier 2001. 


\section{REMERCIEMENTS}

Je tiens d'abord à exprimer ma reconnaissance à ma directrice de recherche, Madame Marta Anadón, Ph.D., professeure-chercheure au Département des sciences de l'éducation et de psychologie de l'Université du Québec à Chicoutimi, sans qui je n'aurais pu mener à terme ce travail. Sa patience, sa disponibilité et sa rigueur scientifique furent pour moi une aide précieuse.

Je veux également remercier toutes les personnes qui, de près ou de loin, m'ont supportée dans la réalisation de cette recherche.

Enfin, j'aimerais remercier ma famille et mes amies qui ont su m'encourager et me convaincre de poursuivre dans les moments difficiles. 


\title{
LISTE DES PRINCIPAUX SIGLES
}

\author{
ADRSIQ : Association des directeurs et responsables de soins infirmiers du Québec \\ AEESIC : Association des enseignantes et des enseignants en soins infirmiers des \\ collèges du Québec \\ AHQ : Association des hôpitaux du Québec \\ AIIC : Association des infirmières et infirmiers du Canada \\ AIPQ : Association des infirmiers de la province de Québec \\ CHSGS : Centre hospitalier de soins généraux et spécialisés \\ CHSLD : Centre hospitalier de soins de longue durée \\ CLSC : Centre local de services communautaires \\ DGEC : Direction générale de l'enseignement collégial \\ FIIQ : Fédération des infirmières et infirmiers du Québec \\ MEQ : Ministère de l'Éducation du Québec \\ OIIQ : Ordre des infirmières et infirmiers du Québec \\ SSC : Suivi systématique de la clientèle \\ UQAC : Université du Québec à Chicoutimi \\ UQAH : Université du Québec à Hull \\ UQAM : Université du Québec à Montréal \\ UQAR : Université du Québec à Rimouski \\ UQAT : Université du Québec en Abitibi-Témiscamingue \\ UQTR : Université du Québec à Trois-Rivières
}




\section{TABLE DES MATIÈRES}

RÉSUMÉ

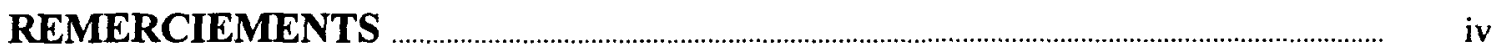

TABLE DES MATIÈRES

LISTE DES PRINCIPAUX SIGLES ……............................................................... v

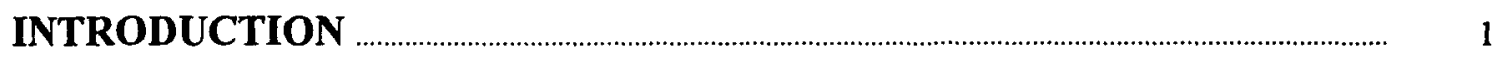

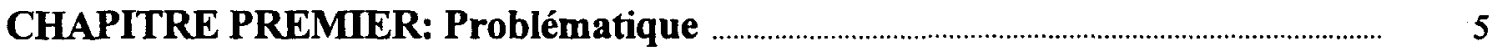

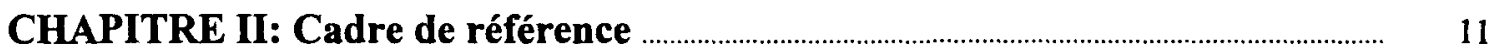

2.1 Demandes sociales ................................................................................................. 11

2.2 Professionnalisation ............................................................................................. 21

2.3 Formation ......................................................................................................... 30

CHAPITRE III: Méthodologie …........................................................................................ 45

3.1 Introduction ................................................................................................... 45

3.2 Constitution du corpus ........................................................................... 50

CHAPITRE IV: Analyse de contenu ...................................................................................... 55

4.1 Contexte social ........................................................................................... 55

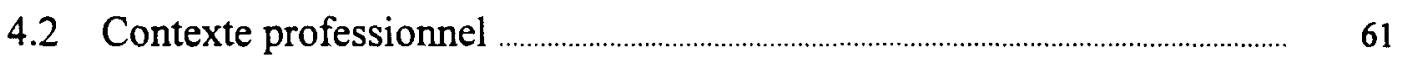

4.3 Contexte de formation ...................................................................................................... 65

4.4 Synthèse des résultats de l'analyse de contenu .................................................. $\quad 79$

CHAPITRE V: Analyse du programme collégial en soins infirmiers élaboré

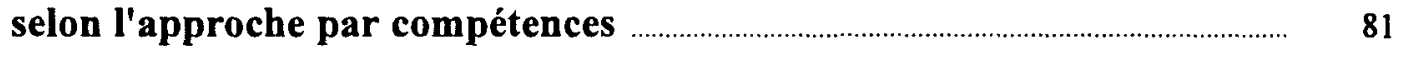

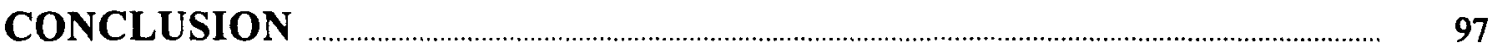

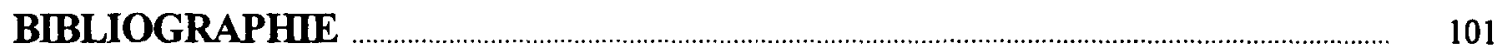




\section{INTRODUCTION}

Encore aujourd'hui, la formation infirmière est au cœur de nombreux débats. Cette fois-ci, deux réformes majeures, celle de la santé et celle de l'éducation, auront un impact majeur sur la formation et la pratique infirmières. Concrètement, plusieurs changements sont survenus dans les établissements autant du réseau de la santé que de celui de l'éducation, suite aux compressions budgétaires imposées par le gouvernement du Québec. En santé, ces compressions supposent l'émergence d'une pratique modifiée pour les infirmières qui devront assumer de nouveaux rôles et distribuer les soins selon de nouvelles approches.

Parallèlement à la réforme de la santé, le ministère de l'Éducation du Québec, par la réforme de l'éducation, propose une révision des programmes de formation pour tous les ordres d'enseignement. Ainsi, en éducation, on mise sur l'approche par compétences pour améliorer les programmes d'études de tous les niveaux d'enseignement y compris le niveau collégial. Dans le cas qui nous préoccupe, celui de la formation en soins infirmiers, le programme collégial est entièrement révisé pour donner jour à un nouveau programme élaboré selon l'approche par compétences et dont l'implantation est prévue pour l'automne 2001. Dans le cadre du présent mémoire, nous cherchons à savoir si la formation collégiale en soins infirmiers permet de répondre aux demandes actuelles en soins infirmiers. Pour ce faire, nous avons recours à deux analyses: d'abord, une analyse de contenu nous permet d'identifier les nouvelles demandes sociales en émergence; ensuite, une ana- 
lyse du «Projet de programme en soins infirmiers» conçu selon l'approche par compétences nous permet d'examiner si ce nouveau programme répond aux demandes sociales identifiées à la suite de l'analyse de contenu.

La réforme de la santé a modifié considérablement les modes de distribution de soins. Le virage ambulatoire, en favorisant l'auto-prise en charge du citoyen, fait émerger une nouvelle pratique infirmière qui fait appel à plusieurs compétences. Les infirmières sont appelées à élargir leur rôle et à travailler davantage dans la communauté. Il semblerait que cette nouvelle réalité suggère l'acquisition de nouvelles compétences pour ces travailleuses de la santé. La formation collégiale en soins infirmiers répond-elle aux nouvelles demandes sociales en soins infirmiers? C'est la question autour de laquelle s'organise notre problématique de recherche.

En effet, c'est précisément à cette question que nous tenterons de répondre à l'aide de deux analyses distinctes: l'une portant sur les documents officiels et l'autre sur le programme d'enseignement collégial en soins infirmiers.

Une analyse de contenu à partir de textes «officiels», produits par divers organismes publics intéressés à la pratique infirmière, nous permet de mettre en relief les différents aspects qui peuvent influencer la formation de l'infirmière. Quant à l'analyse du nouveau programme collégial en soins infirmiers, dont l'implantation est prévue pour l'automne 2001, elle aide à évaluer si les compétences visées permettent de répondre aux demandes actuelles de la population en soins infirmiers.

Dans un premier temps, nous avons lu, sans faire de sélection, plusieurs textes publiés au sujet de la formation infirmière. Au terme de cette première lecture, l'ampleur des 
publications nous a orientée, à sélectionner des textes à partir de certains critères spécifiques. Par la suite, après d'autres lectures, nous avons choisi de procéder à l'analyse de contenu en situant les textes dans trois contextes différents (contexte social, professionnel et de formation) pour en faire ressortir le point de vue de chacun des organismes impliqués dans cette problématique et ce, en relation avec chacun de ces contextes. Concernant l'analyse du nouveau programme collégial en soins infirmiers, le seul document analysé est une copie du projet Soins infirmiers 180.A0. Malgré les limites que nous nous sommes imposée, nous espérons, par ces deux analyses, répondre à notre question de recherche en mettant en relief certains éléments signifiants dans le dossier de la formation infirmière.

Pour faciliter la compréhension et situer le lecteur, nous avons recours à plusieurs sigles dont la liste est placée au début du document, pour identifier les principaux organismes auxquels nous faisons référence. Le premier chapitre nous permet d'introduire les nouvelles demandes en soins infirmiers découlant de la réforme du système de la santé amorcée au début des années 90 par le gouvernement du Québec. C'est dans ce contexte particulier que notre problématique de recherche se justifie. Vient ensuite l'élaboration du cadre de référence dans le deuxième chapitre, que nous divisons selon les mêmes grands contextes: les contextes social, professionnel et de formation. Une courte lecture historique permet d'introduire chacun des contextes et de voir l'évolution qu'il a connue au cours des années. Le troisième chapitre, réservé à la méthodologie de la recherche, présente les critères qui nous amènent à choisir l'analyse de contenu comme méthode de lecture des textes sociaux ainsi que le corpus de textes retenus pour fins d'analyse. C'est au quatrième chapitre que le travail d'analyse est présenté. Les textes sont analysés selon les trois contextes cités précédemment, c'est-à-dire le contexte social, le contexte professionnel et le contexte de formation. Nous rapportons le discours de chacun des organismes 
pour chacun des trois contextes, et nous le mettons en parallèle avec les autres afin de ressortir des éléments de ressemblance ou de dissemblance. Puis, nous faisons une brève critique de ces discours lorsqu'ils nous apparaissent incohérents les uns par rapport aux autres ou tout simplement contradictoires. Nous présentons également les résultats de l'analyse de contenu et le lien avec nos questions de recherche. Nous choisissons, au cinquième chapitre, de faire une courte description de l'approche par compétences, puisqu'un nouveau programme collégial en soins infirmiers élaboré selon cette approche démarrera dès l'automne 2001 dans les 42 collèges de la province offrant la formation en soins infirmiers. Enfin, nous faisons une brève analyse du nouveau programme à partir du document Projet de programme soins infirmiers 180.A0 afin de vérifier s'il répond aux nouvelles demandes sociales en soins infirmiers. 


\section{CHAPITRE PREMIER}

\section{Problématique}

Actuellement, au Québec, la formation permettant l'accès à la pratique infirmière soulève de nombreuses controverses. Le malaise se perçoit autant dans les milieux d'enseignement et de travail qu'au sein même de l'Ordre professionnel des infirmiers et infirmières du Québec (OIIQ). L'ampleur des transformations des réseaux de la santé et de l'éducation a amené l'Ordre des infirmières et infirmiers du Québec à convoquer, en mai 1996, ses membres aux États généraux de la profession d'infirmière ${ }^{1}$ afin de réfléchir collectivement sur l'avenir de la profession infirmière. Aussi, le document Pour soigner notre avenir. Les infirmières à la croisée des chemins a été publié. On y retrouve entre autres les mémoires qui ont été présentés à la commission des États généraux et un abrégé des trois forums thématiques qui ont donné lieu à des échanges animés.

Le 22 novembre 1996, lors d'une réunion provinciale convoquée par l'OIIQ et à laquelle participèrrent les responsables de programmes de formation infirmière de l'ensemble des collèges et des universités du Québec, le nouveau Conseil provincial sur la formation infirmière voit le jour. La création de ce Conseil provincial est, selon la présidente de l'OIIQ, l'expression de la volonté des infirmières telle qu'exprimée lors des États généraux de la profession. En effet, lors des États généraux de la profession, les infirmières

1 Dans ce travail, le terme infirmière désigne aussi celui d'infirmier. 
demandaient «que soit favorisée la formation par la création d'un comité de concertation réunissant les différents intervenants dans un esprit de respect mutuel. Et ce, dans le but d'atteindre l'unicité du programme» que ce soit au collège ou à l'université (OIIQ, 1996, p.121). Ce comité avait comme mandat de favoriser la concertation et d'accroitre la participation des infirmières aux instances décisionnelles en matière de formation (AEESICQ, 1997). Présentement, au Québec, il existe deux voies d'accès à la profession: la formation collégiale et la formation universitaire. D'une part, le programme Soins infirmiers 180.01, présentement en révision, est offert dans 42 collèges à travers la province et, d'autre part, en formation initiale en sciences infirmières est offerte dans quatre des neuf universités québécoises qui offrent une formation en sciences infirmières. Au terme de ces deux formations, les étudiantes de ces deux niveaux sont soumises au même examen de droit de pratique; la réussite de cet examen est obligatoire pour être inscrite au tableau de l'OIIQ. Cette situation amène d'ailleurs de la confusion et du mécontentement étant donné que la durée et le contenu de ces formations sont différents. En effet, la formation collégiale en soins infirmiers est d'une durée de trois ans (six sessions) tandis que la formation universitaire, également de trois ans, est accessible après deux ans d'études collégiales en sciences de la nature ou en sciences humaines. La formation collégiale prépare l'infirmière à donner des soins infirmiers qui relèvent des aspects de la promotion et du recouvrement de la santé à un individu tandis que la formation universitaire prépare l'infirmière à donner des soins infirmiers selon une approche collective (DGEC, 1987). Malgré les distinctions entre les deux programmes de formation, les principaux facteurs au cœur des discussions actuelles sur la formation infirmière au Québec nous semblent étroitement liés aux changements récents apportés dans le système de santé. Le but d'un système de santé est, comme le rapporte Field (1973: cité par Fournier, 1999, p.16) «de combattre les différents problèmes de santé d'une société et de protéger les individus, voire d'améliorer leur capacité à jouer leurs rôles sociaux». Comme le confirme la Direction générale 
de la formation professionnelle et technique, à la base des transformations dans le système de santé, on retrouve un changement de conception de la notion de santé.

Aussi, l'Organisation mondiale de la santé (OMS), au début des années 80, au moment de la crise des finances publiques, a formulé une nouvelle définition de la notion de santé. Longtemps définie comme l'absence de maladie, la santé est de plus en plus associée à la notion de bien-être et fait référence aux conditions de vie de l'individu et de la collectivité (MEQ, 1996). Et, selon l'OIIQ, ce sont les soins infirmiers qui sont le point d'appui de l'infrastructure du système de santé d'un pays (OIIQ, 1989). Dans le même sens, Dallaire (1999, p.50) rapporte que :

[...] les soins infirmiers comptent pour beaucoup dans la vie des personnes qui en reçoivent: ils entretiennent la vie, ils aident à résoudre des problèmes lorsque la vie rencontre des obstacles, ils aident à traverser des situations critiques, ils informent, ils conseillent; bref, ils soutiennent la poursuite des objectifs de santé de la population.

D'ailleurs, Cohen (2000) rapporte que, de tous les intervenants de la santé, les infirmières jouissent de l'appui populaire le plus élevé. De plus, les infirmières contribuent au bon fonctionnement du système de santé et fournissent des efforts visant la réduction des dépenses (Dallaire, 1999). Le ministère de la Santé et des Services Sociaux du Québec (MSSS) adoptait, au début des années 90, de nouvelles orientations visant à satisfaire, d'une part, des objectifs de qualité des services et, d'autre part, des objectifs d'ordre budgétaire. Plus spécifiquement, par la nouvelle réforme ${ }^{2}$ de la Santé et des Services sociaux, le Ministère désire se donner les moyens pour atteindre les objectifs suivants: mieux ré-

2 Une réforme est un changement majeur, souhaitable et valide d'une situation, d'une chose ou d'une institution, d'un état initial à un état projeté. Une réforme s'inscrit nécessairement dans l'optique d'un changement en bien, d'une amélioration d'une situation présente vers une situation projetée. (LEGENDRE, R., 1993. Dictionnaire actuel de l'éducation, $2^{\bullet}$ éd., Montréal: Guérin, p.1100). 
pondre aux besoins changeants de la population, profiter des nouvelles technologies, offrir des services décentralisés plus près des gens et, enfin, mettre l'accent sur la prévention et la promotion de la santé (MSSS, 1997).

Depuis, le système de distribution de soins a subi de multiples transformations; ce qui a eu pour effet d'entraîner des déplacements dans les différentes activités liées au secteur de la santé (Lefebvre et Brûlé, 1997). Certains soins spécialisés seront éventuellement offerts en clinique externe dans des institutions spécialisées, alors que les centres hospitaliers deviendront fort probablement d'immenses centres de soins intensifs ou semi-intensifs, où se prodigueront des soins de plus en plus complexes, faisant appel à une technologie très avancée (Direction générale de la formation professionnelle et technique, 1996). La fermeture de plusieurs établissements et la fusion ou la conversion de certains autres entraînent des coupures de postes et le déplacement du personnel infirmier. Beaulieu (1997, p.19) écrit dans un éditorial bien connu: «les turbulences sont telles que personne, pas même le ministre de la Santé et des Services sociaux, ne peut tracer aujourd'hui un portrait exact du nouveau réseau qui émerge». En plus des changements apportés dans la mission des établissements de santé, on parle de virage ambulatoire, de suivi systématique de la clientèle et de système Info-santé (Lefebvre et Brûlé, 1997). Comme le rapporte la Direction générale de la formation professionnelle et technique (1996), ces changements nécessiteront une très grande collaboration entre le réseau privé et le réseau communautaire. Selon Lefebvre et Brûlé (1997), des répercussions sur la pratique professionnelle des infirmières sont à prévoir. Des changements autant quantitatifs que qualitatifs s'annoncent pour le personnel infirmier. Les infirmières prendront une place de plus en plus grande, que ce soit en milieu hospitalier ou en milieu communautaire. De son côté, Têtu (1995) soutient qu'il s'agit de nouvelles exigences pour la pratique infirmière dans le contexte du service ambulatoire: l'infirmière devra être polyvalente, autonome, 
flexible, utiliser son expertise, augmenter son rôle d'éducatrice et son rôle de coordinatrice des soins. En plus d'être autonomes, les infirmières de demain devront faire preuve de maturité et posséder des valeurs, des attitudes, des connaissances et des habiletés intellectuelles nécessaires à leurs responsabilités qui sont de plus en plus considérables envers la société (Kérouac, 1994). L'intervention des infirmières dans la communauté implique une rencontre plus concrète avec des univers culturels diversifiés, dans des conditions physiques et d'autonomie de pratique différentes de celles des établissements (Fournier, 1999, p.16). Pour Fournier (1999), les infirmières deviendront des ressources pour les gens plutôt que des ressources au service du système de santé et des autres professionnels de la santé, elles auront des fonctions d'éducation, de collaboration et de coordination.

Quant à l'avenir, l'Association des directeurs et responsables des soins infirmiers fait référence à des tendances et orientations qui transforment radicalement les soins infirmiers (ADRSI; 1993). Les tendances identifiées se définissent autour du concept d'évolution: évolution des usagers, évolution du cadre institutionnel, évolution des droits des usagers, évolution des technologies, évolution de la main d'œuvre en soins infirmiers et évolution des réponses sociales aux problèmes de santé.

Nous croyons que tous ces changements amènent les infirmières à assumer de nouveaux rôles dans la société et apportent de nouveaux arguments au processus de professionnalisation qui semble prendre de plus en plus d'ampleur chez ce corps de métier. Étant donné que la formation universitaire est un des critères normatifs de la définition d'une profession, la question qui se pose est de savoir si la formation collégiale peut répondre aux nouvelles demandes sociales en soins infirmiers, c'est-à-dire aux nouvelles orientations du domaine de la santé au Québec. 
Afin de répondre à cette question, il devient nécessaire de mieux connaître les nouvelles orientations du réseau de la santé. Une analyse de contenu, à partir des documents produits par les organismes qui s'intéressent de près ou de loin à la formation des infirmières (MEQ-OIIQ-FIIQ-ADRSI) nous permettra d'identifier et d'analyser ces nouvelles orientations. Puis, dans un deuxième temps, nous porterons un regard sur le nouveau programme collégial en soins infirmiers, révisé selon l'approche par compétences, dont l'implantation est prévue pour l'automne 2001, afin d'examiner si ce dernier permet de répondre aux nouveaux besoins qui semblent orienter la pratique infirmière.

Mais auparavant, nous verrons plus en détail, dans le chapitre réservé au cadre conceptuel, les concepts généraux qui semblent être en lien étroit avec la nouvelle pratique infirmière en émergence. 


\section{CHAPITRE II}

\section{Cadre de référence}

Ce second chapitre prolonge la démarche d'investigation entreprise dans le chapitre précédent, où comme on l'a vu, la question de recherche nous conduit à nous situer autant dans une perspective d'exploration et de description que dans une perspective de vérification. Notre démarche touche à la fois l'identification des nouvelles demandes sociales en soins infirmiers et l'analyse du nouveau programme de formation collégial avec l'objectif d'examiner sa capacité à former un professionnel capable de répondre avec compétence à ces nouvelles demandes de la société.

\subsection{Demandes sociales}

Les discussions actuelles sur la formation infirmière au Québec semblent être en lien direct avec les nouvelles orientations du système de santé. L'émergence d'une nouvelle pratique a amené l'OIIQ et ses membres à réfléchir collectivement sur l'avenir de la profession infirmière au Québec. Les nouveaux objectifs du MSSS, comme le soulignent Lefebvre et Brûlé (1997), auront des répercussions importantes sur la pratique infirmière. En effet, les changements organisationnels amenés par la réforme de notre système de santé ont modifié la mission des établissements et la configuration du réseau (Blouin; 1994). Pour comprendre les changements actuels du système de santé québécois, nous devons retourner au début du $\mathrm{XX}^{\mathrm{e}}$ siècle. 
L'OIIQ (1991) rapporte que jusqu'en 1921 ce sont les communautés religieuses, les familles et les paroissiens qui ont été les responsables des soins aux malades. Puis, à partir de 1921, moment où la Loi de l'assistance publique a été adoptée, l'État commence à jouer un rôle important dans la distribution des services de santé. En 1961, par l'avènement de l'assurance-hospitalisation le gouvernement fédéral vient complètement changer les sources de revenus des hôpitaux (OIIQ, 1991). En effet, c'est à partir de ce moment que la majorité des frais hospitaliers sont assumés par l'État. En 1966, les travaux de la Commission Castonguay-Nepveu ont donné une orientation précise au système et ont conduit à la mise sur pied de nouvelles institutions québécoises dont la Régie de l'assurance-maladie du Québec en 1969. Les principes à la base de cette réforme sont la médecine globale et la pratique d'équipe, la décentralisation du pouvoir de décision vers la communauté, la participation et l'égalité des droits des travailleurs de la santé. Or, il semblerait qu'au lieu de remédier à certaines situations critiques, cette réforme a eu pour effet d'en accentuer quelques-unes. En effet, l'application du régime d'assurance-maladie a favorisé l'escalade des soins curatifs et a créé une plus grande dépendance de la population envers un réseau hospitalier de plus en plus coûteux. Renaud (1981: cité dans Fournier, 1999, p.12) qualifie cette situation de despotisme cognitif: «nos vies deviennent de plus en plus soumises aux commandements et aux visions du monde des élites médicales, dont l'efficacité thérapeutique est d'ailleurs beaucoup moins évidente qu'au moment de la lutte contre les maladies infectieuses».

Près de vingt ans plus tard, précisément en 1985, la santé fait à nouveau l'objet d'une autre commission d'enquête, la Commission Rochon. Au terme de ces travaux, la Commission a réaffirmé la finalité fondamentale du système de services de santé et de services sociaux, à savoir l'amélioration de la santé et du bien-être, et elle a identifié deux conditions préalables: la recherche d'une plus grande équité sociale et le développement 
de l'équilibre social. L'enquête réalisée par la Commission Rochon a permis de constater, entre autres, l'absence d'objectifs et de priorités à long terme, l'insuffisance d'information disponible sur la santé et le bien-être et une démotivation du personnel dans les établissements du réseau. Les recommandations formulées par la Commission visent les orientations suivantes: un système centré sur l'atteinte des résultats; une approche intersectorielle tant aux niveaux local, régional que provincial; la participation des citoyens dans les instances décisionnelles, les instances consultatives et les instances de concertation du système; l'adoption des objectifs et des moyens en fonction des réalités régionales; une approche de la population permettant d'agir sur les facteurs déterminants des problèmes; l'introduction d'un système public sur les plans du financement et du fonctionnement (Potter; 1990) . Depuis la commission Rochon, la situation financière précaire du gouvernement remet en question l'État providence (Fournier, 1999).

Pour sa part, le projet de réforme des services de santé et des services sociaux (Loi 120), rendu public en décembre 1990 par le ministre Marc-Yvan Côté, s'articule autour de trois pôles dont le premier est le respect de la personne et de l'employé du réseau; le deuxième concerne la satisfaction des besoins de ces derniers et, le troisième, la participation active du citoyen et de tous les artisans œuvrant dans le domaine de la santé ainsi que des agents des autres secteurs d'activité (OIIQ, 1991). C'est dans le but de resituer les usagers au cœur du réseau que la nouvelle réforme de la santé et des services sociaux propose neuf orientations ayant pour cible le citoyen consommateur (l'accent est mis sur la reconnaissance et le respect des droits des citoyens); le citoyen décideur (la réforme souhaite que le citoyen soit au cœur du processus de décision) et le citoyen payeur (la réforme propose que la société assume le coût des services et en ait pour son argent). Enfin, les mesures proposées par le projet de Loi 120 entrainent des changements profonds dans l'organisation et dans le fonctionnement du système de santé et des services 
sociaux. Le financement et la mise en place d'une politique de santé permettront à la réforme de prendre son envol. En plus d'amener une transformation du système de santé, la réforme suppose un changement de philosophie. Ainsi, l'accent sera mis sur la prévention et sur les déterminants sociaux de la santé (Villedieu, 1996). Selon Fournier (1999), une intervention sur les maux sociaux s'avère aussi importante, si ce n'est plus, que sur des maladies physiques graves. Les défis de la réforme de santé, comme le rapporte Fournier (1999), peuvent constituer des ouvertures intéressantes pour la profession infirmière en consolidant et en développant certains rôles infirmiers.

En effet, selon Goulet O. et Dallaire (1999), le virage ambulatoire permet une pratique plus autonome et offre plus d'opportunités de travail en soins infirmiers: cliniques de première ligne en CLSC, suivi systématique de clientèles, prise en charge globale de la clientèle dès son arrivée en établissement ou au service Info-santé et la pratique privée. Quelques brèves définitions de ces nouveaux concepts nous apparaissent essentielles afin de mieux faire comprendre comment le rôle de l'infirmière a été modifié.

\section{Virage ambulatoire}

Les services de soins ambulatoires correspondent à des services impliquant un déplacement soit, des personnes qui ont besoin de soins soit, des personnes qui administrent les soins. Donc, il y a une transformation dans la façon de dispenser les services. Le virage ambulatoire se veut une solution de rechange à l'hospitalisation et à l'hébergement (Soins infirmiers Étude préliminaire, MEQ, 1996, p.13-14).

Il implique, comme le rapporte Fournier (1999), le recours aux ressources communautaires et familiales qui, d'ailleurs, selon l'avis du Ministère, est justifié par un coût moindre et par le désir des individus à se prendre en charge. 
Pour Villedieu (1996, p.66),

le virage ambulatoire consiste à ne faire à l'hôpital que ce qu'on ne peut absolument pas faire ailleurs et surtout à accueillir le patient le matin même de l'opération, ou de l'examen ou de l'administration d'un traitement et à le retourner chez lui le soir même.

Doye (1997, p.59), pour sa part, donne la définition suivante:

Processus dynamique visant à ce que certains services de santé et services sociaux dispensés traditionnellement dans un contexte d'hospitalisation ou d'hébergement, soient offerts plus près du milieu de vie naturel du bénéficiaire. Cette démarche doit offrir une plus grande accessibilité aux services dont la prestation se fait par des ressources plus légères. Elle sous-tend la diminution, voire l'évitement du séjour en institution, ceci dans l'assurance d'une qualité et d'une continuité optimales des services, ainsi que d'une sécurité adéquate des bénéficiaires. Le tout doit s'actualiser à un coût social moindre que celui requis antérieurement.

Selon l'étude préliminaire en soins infirmiers: «Il semblerait que les deux secteurs qui pourraient connaître le plus grand développement sont celui du maintien à domicile et celui de la santé mentale» (MEQ, 1996, p.23). Par ailleurs, la capacité du milieu à se prendre en charge, souligne Fournier (1999), représente un élément essentiel à la réussite du virage ambulatoire et s'inscrit dans un mouvement de responsabilisation des individus. L'hôpital est désormais l'espace privilégié du traitement et non plus de la convalescence. D'autres structures doivent prendre la relève en ce qui a trait à l'hébergement, les services de première ligne et les soins de convalescence (Fournier, 1999).

\section{Suivi systématique de la clientèle}

Le Suivi systématique des clientèles (SSC) tire ses origines de l'ingénierie industrielle et de la gestion de projets. Au début il fut appliqué comme approche dans les services de santé et services sociaux dans le but de procurer aux personnes nécessiteuses des ressources de base telles la nourriture, le chauffage et le gîte (Bolduc, 1997, p.6). 
Dans le domaine de la santé, Bolduc (1997) rapporte que c'est au New England Medical Center Hospitals de Boston que les infirmières ont utilisé pour la première fois des outils de cheminement critique pour des bénéficiaires en soins aigus. Au Québec, le contexte de restrictions budgétaires actuel oblige les hôpitaux à recourir à de nouvelles approches pour dispenser les soins. Le suivi systématique de clientèles semble être une alternative de plus en plus proposée car elle propose une gestion différente de l'épisode de soins. Cette nouvelle approche, inscrite dans une perspective élargie, inclut la totalité de l'épisode de soins, depuis l'admission en centre hospitalier jusqu'à la guérison. Bolduc (1997, p.12) la définit comme étant:

La gestion d'épisodes de soins axés vers des résultats à atteindre pour et avec le bénéficiaire, réalisés dans un laps de temps spécifique qui peut varier tant en fonction de l'épisode de soins qu'en fonction du bénéficiaire lui-même, tout en utilisant les ressources disponibles de façon optimale.

Cet auteur précise qu'il s'agit de dispenser les bons soins/services, au bon bénéficiaire et au moment optimal à l'aide d'un plan interdisciplinaire de soins/services. Le suivi systématique de la clientèle doit être envisagé comme un système en évolution et être réévalué périodiquement.

Selon Bower (1995: cité dans Bolduc, 1997, p.11), le suivi systématique est:

Une démarche clinique dont l'emphase est mise sur la responsabilité d'un individu ou d'un groupe d'individus pour: coordonner les soins à un bénéficiaire ou à un groupe de bénéficiaires pendant toute la durée de l'épisode de la maladie; négocier, dispenser et coordonner les soins, les services ainsi que les ressources requises par le bénéficiaire et ses proches; s'assurer de et faciliter l'atteinte d'objectifs de qualité, de soins et de coûts; intervenir à des étapes clés; s'occuper et résoudre les situations présentant un impact négatif sur la qualité et les coûts; et créer des opportunités pour en améliorer la démarche en termes de résultats. 
Cette dernière définition, à notre avis, fait ressortir le rôle élargi que devra tenir l'infirmière dans le contexte de la nouvelle réforme de la santé. Selon Bolduc (1997, p.23), les principaux avantages du SSC pour l'équipe de soins sont:

une pratique professionnelle rehaussée;

une planification des activités de soins améliorée; l'augmentation de l'esprit de collaboration et de coopération; la diminution des oublis et/ou erreurs; l'identification du rôle de chacun dans l'épisode de soins; le développement des ressources humaines.

\section{Intervention de première ligne}

Il est précisé à l'article 54 du projet de Loi 120, sanctionné le 4 septembre 1991, «Loi sur les services de santé et les services sociaux et modifiant diverses dispositions législatives», que:

La mission d'un centre local de services communautaires est d'offrir, en première ligne, à la population du territoire qu'il dessert, des services de santé et des services sociaux courants, de nature préventive ou curative, de réadaptation ou de réinsertion. À cette fin, l'établissement qui exploite un tel centre s'assure que les personnes qui requièrent de tels services pour elles-mêmes ou pour leurs familles soient rejointes, que leurs besoins soient évalués et que les services requis leur soient offerts à l'intérieur de ses installations ou dans leur milieu de vie, à l'école, au travail ou à domicile ou, si nécessaire, s'assurer qu'elles soient dirigées vers les centres, les organismes ou les personnes les plus aptes à leur venir en aide (Doyon, 1997, p.47).

Les trois éléments distinctifs d'une approche de première ligne sont: un territoire bien défini, une gamme spécifique de services et un concept particulier de qualité (Fédération des CLSC du Québec, 1995). Les principales fonctions de ce service sont : l'Accueil/Évaluation, l'Information/Conseil et l'Orientation/Référence (Dunnigan, 1996). En plus de renseigner sur les services sociaux et de santé disponibles, par un accès télépho- 
nique continu 24 heures par jour 7 jours sur 7 visant à faciliter l'orientation des citoyens dans le réseau de la santé et des services sociaux, le service fournit des conseils professionnels pour les situations qui ne nécessitent pas d'intervention spécialisée immédiate. De plus, ce service, comme le spécifie Dunnigan (1996, p.9), doit privilégier «une approche globale, laquelle intègre à la fois les dimensions biologiques, psychologiques et sociales, devra être axé sur le maintien ou le renforcement de l'autonomie des personnes et être en mesure de répondre à toutes les problématiques de nature sociosanitaire». Il semble que l'expertise et la compétence du personnel aient été identifiées comme des facteurs de réussite de ce service (Dunnigan, 1996). Le MSSS (1997) précise que le rôle de l'infirmière en intervention de première ligne est de conseiller et orienter le client vers le service qui répondra le mieux à ses besoins. Doye (1997, p.58) identifie les caractéristiques d'une intervention de première ligne en CLSC comme étant les suivantes:

1. légère, volontaire, accessible à toute la population.

2. de prévention et/ou de résolution de problème.

3. individuelle, de groupe ou collective.

4. fondée sur une vision globale des problèmes.

5. réalisée par un ou des membres d'équipe multidisciplinaire.

6. suivant une approche communautaire.

7. en partenariat avec les instances pertinentes des réseaux (publics, communautaires, sociaux-sanitaires).

8. dans une perspective de responsabilisation des individus et des communautés.

9. dans une perspective de maintien ou de réinsertion dans le milieu naturel.

10. peu coûteuse.

Le virage ambulatoire, l'intervention en première ligne et le suivi systématique de la clientèle sont des modes de distribution de soins implantés dans le but d'atteindre les objectifs de la réforme. 
Les brèves définitions que nous avons rapportées sur ces nouveaux concepts semblent essentielles pour mieux comprendre les modifications du rôle de l'infirmière dans un avenir rapproché. Il ne s'agit pas ici de faire l'analyse ni la critique de la réforme mais de faire ressortir les éléments qui nous semblent liés de près ou de loin à la pratique infirmière. Comme le souligne Potter (1990), les soins infirmiers sont une composante essentielle de la société, qui évoluent donc avec elle, et dont l'objectif a toujours été de satisfaire les besoins en matière de santé. En effet, au Québec, jusqu'au début du XX' $\mathrm{XX}^{\mathrm{e}}$ siècle, la responsabilité des soins aux malades revenait à la famille, à la paroisse et aux communautés religieuses (OIIQ, 1991). C'est à partir de 1921, année de l'adoption de la Loi de l'assistance publique qui assure les frais d'hospitalisation, que l'État commence à jouer un rôle plus déterminant dans la distribution des services de santé. Le XIX ${ }^{\mathrm{e}}$ siècle marque aussi la naissance des écoles d'infirmières. Les élèves apprennent à soigner, à donner les premiers soins, à panser et à surveiller l'administration des remèdes (Riopelle et LeducLalonde, 1982). L'OIIQ (1991) rapporte qu'en plus d'être des femmes dévouées, serviles, charitables et conciliantes, les infirmières de cette époque font preuve d'une grande soumission et sont dépourvues de toute autonomie dans l'exécution des tâches du métier. Ainsi, les interventions, qu'elles peuvent faire, sont déterminées par les politiques de l'hôpital, les prescriptions du médecin et les directives de l'infirmière responsable. Les infirmières de l'époque jouent un rôle passif; la profession étant définie, à cette époque, principalement par les médecins et les communautés religieuses.

D'ailleurs, l'histoire des femmes dans la société a aussi influencé la pratique infirmière au Québec. En effet, c'est à partir des années 60, que des changements majeurs se produisent chez les infirmières; changements provoqués par l'adoption de nouvelles valeurs par des femmes qui désirent se détacher de plus en plus des rôles serviles qu'elles ont endossés pendant de nombreuses années. Ainsi, ces changements se caractérisent par 
la recherche de l'autonomie. Par ailleurs, les soins infirmiers comme d'autres savoirs sont inscrits dans le mouvement scientifique, nécessitant l'acquisition de connaissances de plus en plus spécialisées et de plus en plus complexes (Riopelle et Leduc-Lalonde, 1982). Ce vent de changement, malheureusement, ne semble pas atteindre les autorités gouvernementales de l'époque. En effet, malgré les nombreux progrès réalisés par la médecine au cours des dernières années, au lieu d'améliorer la santé et de prévenir la maladie, il semblerait, selon l'OIIQ (1989), que l'essentiel des budgets de santé ait été investi dans les centres hospitaliers où on traite des maladies aiguës.

À l'entrée du troisième millénaire, Trofino (1995: cité par Fournier, 1999) affirme que «l'intérêt pour le bien-être plutôt que pour la maladie rend nécessaire une grande sensibilisation des malades externes à la promotion de la santé et du bien-être». De plus, selon le même auteur, les infirmières assureront une précieuse continuité des soins, en gérant les périodes critiques de maladie et en favorisant le bien-être pour hâter la sortie de l'hôpital, en soutenant les malades externes et en jouant le rôle d'éducatrices sanitaires dans les entreprises, dans les écoles ou dans les domiciles des malades. Fournier (1999) qualifie «d'ouvertures intéressantes» les défis qu'impose la réforme du système de santé à la profession infirmière. En effet, comme nous l'avons déjà précisé, les nouveaux modes de distribution de soins réfèrent aux concepts d'autonomie et de responsabilité professionnelles; concepts qui doivent être compris dans un processus de professionnalisation.

Afin de mieux comprendre ce processus de professionnalisation et de situer les soins infirmiers et leur formation, il semble pertinent de se pencher sur la problématique de la professionnalisation. 


\subsection{Professionnalisation}

Larouche (1987, p.65) rapporte que la notion de professionnalisation permet de répondre aux interrogations suivantes: premièrement, quel cheminement doit généralement suivre une occupation qui aspire au titre de profession? et deuxièmement, à quel niveau d'évolution se situe une occupation qui se professionnalise ou, quelles sont les étapes à franchir? Etzioni (1969: cité dans Larouche, 1997, p.76) apporte des éléments de réponse à ces questions. Il soutient qu'une occupation en voie de se professionnaliser doit être en mesure de répondre à des caractéristiques «naturelles et nécessaires» à toute profession: un ensemble de connaissances abstraites, un idéal de service et les traits spécifiques qui caractérisent le comportement des professionnels. Ces traits spécifiques sont les suivants:

Un revenu élevé, un haut niveau de prestige et d'influence, des exigences éducationnelles élevées, une autonomie professionnelle, un permis de pratique, l'engagement envers la profession, le désir des membres de demeurer au sein de la profession, la présence d'un code de déontologie, l'existence d'un certain monopole sur des tâches à caractère professionnel, l'existence d'une cohésion à l'intérieur de la communauté professionnelle ainsi que le fait que les recrues puissent vivre des expériences de socialisation intensive.

Selon Poletti (1993: cité dans Carpenito, 1997, p.XII):

Ce qui caractérise une profession, ce qui la différencie d'une occupation, c'est la capacité de celui ou de celle qui l'exerce d'expliciter son action, de la baser sur un processus de pensée, sur une réflexion précise, sur des données scientifiques. Être professionnel, c'est savoir exactement ce que l'on fait, pourquoi on le fait et comment l'on pourra vérifier le résultat de ses actions.

Pour Larouche (1987, p.91), le contrôle que les membres possèdent sur leur propre travail et sur le devenir de leur occupation est un des facteurs essentiels qui exerce une 
influence sur le processus de professionnalisation. D'ailleurs, Etzioni (cité dans Anadón, 1999) fait référence à deux types de professions: celles dites à part entière et celles considérées comme semi-professions. Pour cet auteur, les infirmières sont des semi-professionnelles car elles répondent aux caractéristiques suivantes: une forte proportion des membres de sexe féminin, présence d'une image professionnelle en étant conscientes qu'elles ne sont pas totalement qualifiées ni socialement reconnues; la formation est plus courte et moins spécialisée; les membres ont moins d'autonomie, leurs droits et pouvoirs sont moins établis que chez les professions à part entière. En résumé, selon Etzioni (cité dans Anadón, 1998), les infirmières sont des semi-professionnelles car elles n'ont pas de formation universitaire obligatoire au droit de pratique et elles ne sont pas socialement reconnues comme professionnelles même si elles donnent une image professionnelle. Pour d'autres auteurs, il semble que le statut de professionnel soit acquis par les infirmières. En effet, pour Carpenito $(1997$, p.1), la reconnaissance du statut professionnel semble être chose faite: «le cheminement vers la reconnaissance universelle des soins infirmiers en tant que profession fut tumultueux». De plus, selon Carpenito (1997, p.1), «lorsqu'un groupe exerçant une activité cherche à revendiquer un statut professionnel, il doit répondre à certains critères: un enseignement universitaire approfondi, un corpus de connaissances unique, une orientation des services vers autrui, un corps professionnel, une autonomie et une autoréglementation». Pour Doenges (1996), il semble que les infirmières aient lutté pendant de nombreuses années pour obtenir le statut professionnel. Selon cette auteure, ce sont les recherches menées par un groupe d'infirmières, dans le but de déterminer les problèmes des patients relevant de leur champ de compétence pour en faire un diagnostic infirmier, qui ont permis aux infirmières d'obtenir ce statut.

Une autre étude (Anadón, 1999) rapporte que depuis quelques années les sociologues du travail ont abandonné les critères externes pour définir une profession et abor- 
dent la professionnalisation comme étant un processus dynamique qui se donne dans un continuum, même s'il n'y a pas de consensus sur les différentes étapes qui le composent. Anadón (1999, p.7) précise que, selon les travaux de Jenkins (1970), six éléments sont obligatoires à tout processus de professionnalisation:

- l'élément structurel fait référence à la spécialisation, à la centralisation de l'autorité, aux mécanismes de sanctions ainsi qu'à la standardisation.

- l'élément contextuel fait référence à la taille du groupe, aux ressources et au réseau de relations des membres ainsi qu'aux dimensions spatio-temporelles.

- les objectifs et règles du groupe font référence à la composante activité.

- l'élément éducationnel fait référence aux préalables exigés ainsi qu'à la durée et aux coûts de formation.

- la composante idéologique réfère à l'implication des membres, à l'identité du groupe et au sentiment d'appartenance, au statut et au processus de socialisation des membres.

- l'élément comportement repose sur les codes de conduite et sur l'évaluation des membres.

Pourtant, les infirmières sont considérées comme des professionnelles de la santé à part entière, même si leur corps professionnel ne répond pas entièrement aux caractéristiques jugées essentielles à une profession. En effet, les infirmières jouent un rôle important dans le système de santé de notre société par l'activité qu'elles exercent. Prenant en compte les critères élaborés par Jenkins, nous pouvons situer la reconnaissance professionnelle des infirmières. Ainsi, la formation d'une corporation professionnelle, dès le début des années 20 et dont le rôle principal consiste à assurer la protection du public en contrôlant l'accès à l'exercice de la profession et au respect de la discipline, fait référence, selon nous, aux mécanismes de sanctions et de standardisation de l'élément structurel de Jenkins. L'OIIQ constitue la corporation professionnelle dont les effectifs sont parmi les plus élevés au Québec (au-delà de 60,000 membres et 13 corporations régionales), ce qui répond à l'élément contextuel. Les normes de compétence de la profession ainsi que les perspectives de l'exercice de la profession d'infirmière font référence à la composante ac- 
tivité puisqu'elles définissent la pratique infirmière. L'élément éducationnel faisant référence à la durée de la formation constitue, selon nous, le principal obstacle à la reconnaissance professionnelle de l'infirmière, la formation de base universitaire n'étant pas obligatoire à la pratique professionnelle au Québec, contrairement aux autres professionnels de la santé. Enfin, le rôle important que jouent les infirmières dans le système de santé d'une société par l'activité qu'elles exercent, répond à la composante idéologique tandis que les comités de discipline et d'inspection professionnelle de l'OIIQ font référence à l'élément comportement, tel que défini par Jenkins. Ces éléments nous portent à affirmer que les infirmières répondent, hormis la formation, aux critères normatifs ou dit autrement, aux exigences obligatoires de reconnaissance d'une profession.

Le problème au Québec, c'est que le Code des professions reconnaît 43 professions regroupant des métiers aussi divers que podiatre, infirmière auxiliaire, comptable, médecine, etc. (Goulet, O., 1999). Ces professionnels, selon Goulet, O. (1999), n'ont pas tous le même degré de formation, les mêmes responsabilités, la même reconnaissance, mais, sont tous légitimés par l'État par le biais du Code des professions.

Dans le cas particulier des soins infirmiers, St-Amour et al. (1987) situent le «rôle professionnel» de l'infirmière autour de quatre composantes: structurelle, axiologique, éthique et légale. La composante structurelle se rapporte aux relations entre les individus, les personnes et les milieux de formation et de travail tandis que la composante axiologique fait référence au processus de valorisation et à la clarification des valeurs. Les composantes éthiques et légales font référence à des règles de conduite menant au respect de la dignité humaine et à la notion de responsabilité professionnelle. D'autres auteurs, Singleton et Nail (1984: cité dans St-Amour et al., 1987), associent la notion de responsabilité professionnelle à celle d'autonomie. Comme le souligne Goulet, O. (1999, p.3), «la 
professionnalisation n'est pas uniquement visée en raison du statut social qu'elle implique mais parce qu'elle valorise le savoir, la responsabilité, l'autonomie et l'engagement social». Goulet, O. (1999, p.142) rapporte que certains indicateurs permettent de vérifier l'imputabilité d'une profession: «l'enregistrement qui détermine le droit de pratique, la certification de certaines compétences particulières, la surveillance de la formation et le code de déontologie».

Chez les infirmières, un mouvement de professionnalisation semble prendre de plus en plus d'ampleur. Blondeau (1986) rapporte les principales étapes de ce mouvement qui date du début du XIX $\mathrm{X}^{\mathrm{e}}$ siècle. Elle affirme qu'à ses débuts le service infirmier se caractérisait surtout par sa dimension morale et que, depuis, il poursuit une trajectoire ascendante de professionnalisation. Au début du siècle dernier, il semble que le respect de la compétence sont très aléatoire car quiconque pouvait revêtir le costume emblématique de l'infirmière. On parle d'incompétence et même de charlatanisme pour décrire cette époque (Germain, 1985). D'ailleurs, c'est pour se protéger de telles situations que se fondent, à partir des premières années du XXe siècle (1908), les corporations ${ }^{3}$. Comme le souligne Petitat (1989), c'est au tournant du siècle que la vague professionnaliste prend sa source dans un nouveau corps de savoir et que les écoles d'infirmières se portent garantes d'une formation scientifique. Désormais, les diplômées des écoles agréées porteront un titre qui leur sera réservé. Au Canada, ce sont les infirmières de l'Ontario qui ont été les premières diplômées à obtenir la protection de leur titre en 1908, alors qu'au Québec, des dispositions semblables ne seront adoptées qu'en 1920 (Petitat, 1989).

3 Ensemble de personnes exerçant la même profession (Le Petit Larousse illustré (1993), Paris: Éditions Larousse, p.275). 
Chez les infirmières du Québec, Lalancette (1993) rapporte d'autres étapes historiques importantes du processus de professionnalisation chez les infirmières:

1917: les infirmières laïques de Montréal manifestent leur désir de se regrouper pour partager leurs préoccupations dans le but de hausser le niveau de soins;

1920: première loi concernant la profession d'infirmière adoptée par l'Assemblée législative de la province de Québec: Loi constituant en corporation l'Association des gardes-malades enregistrées de la province de Québec (10, George V, chapitre 141). Le titre réservé par la loi: gardes-malades enregistrées;

1938: la registraire de l'association recommande qu'un code d'éthique soit préparé;

1946: à partir de ce moment une loi : Loi concernant l'Association des infirmières de la province de Québec (10, George V, chapitre 88) régit la profession infirmière. Dorénavant l'obtention d'un permis de pratique est nécessaire à la pratique;

1974: la proclamation du Code des professions et la refonte de la Loi des infirmières et infirmiers reconfirment la profession et l'exercice exclusif et définissent l'acte infirmier (p.93-113).

Cette réorganisation du système professionnel, entrée en vigueur en 1974, a été l'occasion pour le législateur de reconfirmer le rôle professionnel de l'infirmière en définissant expressément à l'article 36 de la nouvelle Loi sur les infirmières et les infirmiers, leurs activités professionnelles (OIIQ, 1987, p.12). L'acte infirmier est défini à l'article 36 de la Loi des infirmières et infirmiers:

Constitue l'exercice de la profession d'infirmière ou d'infirmier tout acte qui a pour objet d'identifier les besoins de santé des personnes, de contribuer aux méthodes de diagnostic, de prodiguer et de contrôler les soins infirmiers que requièrent la promotion de la santé, la prévention de la maladie, le traitement et la réadaptation, ainsi que le fait de prodiguer des soins selon une ordonnance médicale.

Si l'on porte une attention particulière à cette définition, on peut observer deux éléments distincts, c'est-à-dire la reconnaissance de deux rôles: l'un d'autonomie (identifier 
les besoins de santé, prodiguer et contrôler les soins infirmiers) et l'autre de collaboration (contribuer aux méthodes de diagnostic et prodiguer des soins selon une ordonnance médicale) dans la pratique infirmière. Si la notion de collaboration fait référence à des actes délégués, la notion d'autonomie, comme le rapporte Goulet, O. (1999), se rattache toujours à la définition d'une profession. La référence fréquente à l'autonomie dans les textes que nous avons analysés nous fait prendre conscience de l'importance de ce concept dans un contexte de professionnalisation. C'est pour cette raison que nous avons choisi de rapporter les définitions de quelques auteurs se rapportant à cette notion.

Pour Singleton et Nail (1984: cité dans St-Amour et al., 1987, p.70), l'autonomie se définit comme étant «la capacité de prendre des décisions et d'agir de façon responsable dans l'intérêt de soi et du client». Pôlet-Masset (1993, p.15) fait plutôt référence à un concept floue à la base du fondement de l'existence de tout individu. Cette auteure affirme que dans un axe dépendance-indépendance, «l'autonomie consiste à assumer ses dépendances et évoluer vers une certaine indépendance » et elle ajoute que "l'autonomie n'est pas l'indépendance mais l'interdépendance, la reconnaissance, la prise en compte de la réalité et de l'existence d'autrui». Selon Thinès et Lempereur (1984: cité dans PôletMasset, 1993, p.15), l'autonomie est la «qualité dynamique de l'individu humain qui se singularise et assume son être propre dans sa rencontre avec autrui en y conservant son intégrité».

Pour sa part, l'Ordre professionnel des infirmières et infirmiers du Québec donne une définition très large du concept d'autonomie. En effet, l'OIIQ rapporte «qu'indépendamment du domaine, l'autonomie suppose la liberté, l'indépendance et la responsabilité de prendre des décisions et de déterminer les règles auxquelles on se soumet» (1989, 
p.29). L'autonomie, en plus d'être une valeur recherchée, est la condition essentielle d'une pratique infirmière «professionnelle» (Goulet, O. 1999).

Pour les infirmières, c'est dans la liberté de prendre des décisions, sans être sous la dépendance des médecins, que se situe la clé de l'autonomie (Kelly et Joël, 1995: cité dans Goulet, O. 1999). Selon Goulet, O. (1999), la turbulence qui secoue actuellement le domaine de la santé, ouvre la voie à la consolidation de l'autonomie pour les infirmières.

Ce bref passage réservé au concept d'autonomie nous semblait essentiel avant de décrire les nombreuses étapes du mouvement de professionnalisation des infirmières du Québec.

Au Québec, depuis 1974, le regroupement des infirmières et infirmiers porte le nom d'Ordre des infirmières et infirmiers du Québec (OIIQ). Les membres doivent se soumettre aux règles et procédures du Code des professions afin d'assurer la sécurité du public. L'OIIQ doit donc déterminer les critères d'exigence permettant l'obtention d'un permis de pratique ainsi que l'inscription au tableau de l'Ordre, de préciser les conditions essentielles permettant d'accéder au marché du travail, d'adopter un Code de déontologie qui précise les devoirs et obligations de l'infirmière envers le public et la profession, de surveiller l'exercice de ses membres par la création d'un comité d'inspection professionnelle et enfin, de contrôler la conduite de ses membres et faire part au Comité de discipline de toute dérogation (O.I.I.Q, 1993).

L'Association des directeurs et responsables des soins infirmiers du Québec a noté des signes concrets qui indiquent que la profession est plus valorisée (1993). En effet, l'amélioration dans l'échelle des salaires, la création d'un conseil des infirmières et infirmiers (CII) par la Loi sur la santé et les services sociaux ainsi que la reconnaissance de la 
valeur des infirmières par la population, ont contribué à rehausser l'image professionnelle de l'infirmière. Pour Blondeau (1999), les deux grandes influences, qui ont marqué l'évolution professionnelle, sont les composantes éthique et scientifique. Comme le rapporte Têtu (1995), les responsabilités de l'infirmière envers la société sont de plus en plus considérables et les transformations du réseau de la santé exigent d'elles, en plus de la polyvalence, de l'autonomie et de la flexibilité, l'acquisition de nouvelles compétences pour la pratique professionnelle.

Comme les compétences s'acquièrent en cours de formation, il semble pertinent de faire une lecture historique de la formation infirmière au Québec. De plus, il semble que la formation a toujours été au cœur des préoccupations des tenants de la professionnalisation de la discipline infirmière (Goulet, O., 1999). En effet, Cohen (2000) rapporte, qu'en Angleterre, au milieu du siècle dernier, une réforme du nursing prenait naissance dans le but de revaloriser le métier d'infirmière et qu'un programme de formation professionnelle rigoureux était élaboré par Florence Nightingale. L'ouverture de la première école d'infirmières de Londres marque le début d'une stratégie de professionnalisation du métier d'infirmière (Cohen, 2000). Cette réforme va s'étendre à toute l'Europe et à l'Amérique et entraînera l'ouverture de 70 écoles d'infirmières à travers le Canada (Cohen, 2000).

$\mathrm{Au} \mathrm{XXI}{ }^{\mathrm{e}}$ siècle, la formation infirmière, par la multiplicité des voies d'entrée, est au cœur de la crise d'identité de la profession infirmière (Korniewicz et Palmer, 1997: cités dans Goulet, O., 1999). Cohen (2000) note un manque de consensus dans la communauté; pour les uns, les soins infirmiers relèvent d'une spécialité et nécessitent une formation universitaire, alors que pour les autres, la formation collégiale suffit à assurer les soins infirmiers généraux en milieu hospitalier et à fournir le nombre d'infirmières nécessaire afin de répondre à la demande. De plus, elle rapporte qu'il faudra uniformiser la forma- 
tion des infirmières pour promouvoir l'autonomie professionnelle. Selon Korniewicz et Palmerm (1997: cités dans Goulet, O., 1999), la segmentation de l'activité des soins infirmiers découle, entre autres, des niveaux de formation différents qui mènent au même droit de pratique. D'où la pertinence de se rappeler brièvement les moments qui ont marqué la formation infirmière au Québec.

\subsection{Formation}

La formation infirmière a, d'ores et déjà, fait couler beaucoup d'encre et a suscité plusieurs débats, comme nous venons de le mentionner dans la section précédente. Une courte lecture historique nous permettra de souligner quelques étapes qui ont marqué la formation infirmière. Nous ne prétendons pas, ici, retracer fidèlement l'histoire de la formation, mais plutôt de souligner quelques étapes qui nous semblent significatives dans l'évolution de cette profession.

Blondeau (1986) rapporte, qu'à ses débuts, le service infirmier se caractérisait par

une dimension morale. À cette époque, les veuves ou les célibataires, appelées diaconesses, se joignaient au clergé pour secourir les malheureux. Le travail des femmes était perçu comme un ensemble de tâches routinières pouvant être accomplies par n'importe qui et ne requérant qu'une courte formation (Parkin, 1995: cité dans Goulet, O., 1999). Les femmes, pensait-on, n'étaient pas biologiquement aptes aux études et n'avaient pas la résistance nécessaire pour entreprendre des études sérieuses (Baumgart et Larsen, 1992: cités dans Goulet, O., 1999). Par la suite, les communautés religieuses ont assuré les soins des malades. Ces bâtisseuses de la première heure ont été des éducatrices prodigues de leur savoir (OIIQ, http://www.oiiq.org/ordre/role.html). Ce sont elles qui, au cours des premières décennies du $\mathrm{XX}^{\mathrm{e}}$ siècle, recrutent les premières infirmières laïques (Blondeau, 1986). 
Comme le rapporte l'OIIQ (http://www.oilg.org/ordre/role.html) : n'entre pas qui veut dans ces écoles. Les responsables de l'admission sélectionnent les candidates selon des critères bien précis, dont la scolarité, l'état de santé et l'excellence de la réputation. La formation, axée sur un modèle médical, visait le développement des qualités personnelles, l'apprentissage de l'altruisme, du don de soi et de l'engagement plutôt que l'acquisition de connaissances et le développement de la capacité de penser et de raisonner (Baumgart et Larsen, 1992: cités dans Goulet, O., 1999). Selon Mundt (1990: cité dans Goulet, O., 1999) ce modèle affecte encore la formation d'aujourd'hui puisque la plus valorisée semble être celle qui prépare à prodiguer des soins.

Les premières études et enquêtes sur la formation donnée aux infirmières ont eu lieu après les années vingt (Germain, 1985). En effet, ce n'est qu'après 1929, suite à une enquête dirigée par le docteur Weir, que les premières réformes débutèrent. La Commission Weir, fut mandatée par les associations d'infirmières canadiennes et provinciales, pour faire enquête sur la formation des infirmières au Canada (Cohen, 2000). La Commission proposa une réduction du pouvoir des administrations hospitalières sur les écoles d'infirmières et que soit revue la sélection des candidates (Cohen, 2000). A partir de cette époque, on souhaite élever les normes d'admission, hausser les qualifications des enseignantes, exiger plus de rigueur scientifique et uniformiser les normes autour d'un minimum plus strict (Germain, 1985). En 1932, Weir (OIIQ, 1982, p.12) écrivit:

Il est probable que la solution la plus satisfaisante aux problèmes de la formation en nursing, comme pour la formation légale, médicale ou autre, ne peut être offerte que par l'université, laquelle possède les meilleures conditions aux plans financement, personnel, équipement pour exercer un sain leadership et servir de réservoir d'idées en matière d'éducation. 
Selon cet auteur, le milieu universitaire, caractérisé par la recherche et la production des connaissances, peut donner à l'étude des problèmes de nursing une vraie dignité et permettre d'attirer un meilleur type de clientèle étudiante. Ainsi, la formation en nursing devrait aussi faire partie des objectifs de l'université (Weir: cité dans l'OIIQ, 1982).

Pourtant, jusqu'en 1944, les infirmières pouvaient être admises dans les écoles des hôpitaux même si elles n'avaient qu'une neuvième année (Goulet, 1993); ce qui est bien loin de la formation universitaire. En 1945, une seconde enquête est commandée par l'Association des infirmières de la province de Québec (AIPQ) afin de faire le point sur la formation des infirmières. Des modifications seront apportées au programme afin de répondre aux nouveaux besoins: des ajouts de stages en psychiatrie et en hygiène publique et des cours de sociologie et de psychologie (Cohen, 2000). C'est au nouveau ministre de l'Éducation que l'AIPQ présentait en 1960, trente ans après le rapport Weir, un mémoire dans lequel elle recommandait que l'enseignement du cours d'infirmière soit confié au ministère de l'Éducation (Desjardins, 1970). L'année 1960 marqua un bouleversement sans précédent dans le domaine de l'éducation. En effet, comme le rapporte Desjardins (1970), c'est à cette époque qu'une enquête royale sur l'enseignement, la Commission Parent, fut instituée. Cette Commission, souligne Lambert (1993), fit des recommandations pour développer l'enseignement post-secondaire au Québec. En effet, ce sont les recommandations du rapport Parent qui allaient transformer le système scolaire du Québec et servir de tremplin pour le premier programme d'études collégial en soins infirmiers (Lambert, 1993).

Pour sa part, Goulet, O. (1999) rapporte que deux commissions d'enquête (Weir, 1932 et Hall, 1964) ont permis de faire ressortir les lacunes de la formation propre aux écoles d'infirmières d'hôpital. L'insuffisance de la formation générale des élèves, ainsi 
que le manque de formation dans certains domaines comme les soins psychiatriques et le manque de formation des enseignantes sont des carences rapportées par ces commissions (Goulet, O., 1999).

Au Québec, il faudra attendre jusqu'en 1970, avec l'avènement des cégeps, avant que la formation infirmière soit prise en charge par le ministère de l'Éducation (Goulet, O., 1999). Lambert (1993) identifie trois événements ayant influencé la formation infirmière de cette époque : la réalité sociopolitique du Québec des années 60, les réactions du milieu infirmier aux réformes proposées quant à l'enseignement des soins infirmiers et enfin, une recherche-action américaine qui s'est produite au cours des années 50 . La Commission royale d'enquête sur l'enseignement créée en 1961 a permis le dépôt du rapport Parent publié entre 1963 et 1965 (Lambert, 1993). Le 29 juin 1967, la loi des collèges fut promulguée et en août de la même année, les cégeps accueillent les premiers étudiants (Lambert, 1993). Goulet, O. (1999) rapporte que c'est le passage à l'enseignement collégial qui a favorisé la consolidation de la formation infirmière, en rendant accessible à un plus grande nombre la formation générale et scientifique. Avant, rapporte Goulet, $O$. (1993), le cours d'infirmière des hôpitaux ne donnait droit à aucune reconnaissance académique de niveau secondaire ou post-secondaire. L'AIPQ se réjouit de cette intégration des écoles d'infirmières au sein du ministère de l'Éducation car elle permettait de changer la formation infirmière pour mieux répondre aux besoins de l'époque (Lambert, 1993). L'AIPQ doit dorénavant partager ses privilèges avec d'autres intervenants: l'État, les écoles publiques, les enseignantes, les syndicats et les fonctionnaires (Cohen, 2000). De plus, cette période d'adaptation fut très difficile car il s'agissait d'un autre système d'enseignement où on devait travailler en fonction d'objectifs prédéterminés. Ceci amena beaucoup de critiques de l'Association des infirmières de la province de Québec (AIPQ) et des milieux cliniques puisque les diplômées n'étaient pas compétentes dans l'immédiat 
(Lambert, 1993). Il semble, comme le rapporte Lambert (1993), qu'il était assez difficile, avec l'ancien modèle de formation des infirmières sous la gouverne des hôpitaux, de distinguer les aspects qui relevaient de la formation de ceux qui visaient à assurer les services de l'établissement.

Il faut souligner qu'à cette même époque, entre 1960 et 1967, les universités implantaient les programmes de formation infirmière. Ces programmes permettaient d'accéder à une formation scientifique renforcée, aux concepts de la discipline infirmière, au développement de l'autonomie intellectuelle, au jugement critique et à la recherche (Goulet, O.). C'est également en 1967 , que le premier programme d'enseignement collégial pour la formation des infirmières fut élaboré par des enseignantes. Au début de la formation collégiale, le programme d'études pour la formation des infirmières portait le nom de Sciences infirmières (Lambert, 1993). Goulet rapporte que le développement de la formation collégiale en soins infirmiers s'inscrit dans une phase où bon nombre d'attitudes et de valeurs qui avaient prévalu jusqu'alors vont désormais être remises en question. Deux stades divisent le développement de la formation collégiale en soins infirmiers: le premier se situe entre 1970 et 1977 et fait référence au passage de la culture hospitalière (infirmières ayant reçu une formation traditionnelle) à celui de la culture collégiale (expériences planifiées et organisées en fonction d'objectifs prédéterminés) et le deuxième stade, qui dure dix ans, marque le début d'une démarche de révision du programme d'études (Lambert, 1993).

En 1976, un comité était mis sur pied par le ministère de l'Éducation, le Comité Rodger, ayant pour mandat d'analyser la situation d'ensemble des programmes en soins infirmiers (FIIQ, 1995). La FIIQ (1995, p.6) rapporte que «le Comité conclut que les cégeps, après réaménagement sont en mesure d'offrir la qualité de programme désirée par 
tous et recommande que le niveau collégial soit le seul niveau à offrir la formation de base en nursing et que le programme de formation se répartisse sur une période de quatre ans».

En 1977, l'OIIQ, en plus de proposer un plan de développement de la formation infirmière au Québec, réaffirme que la formation de base doit se poursuivre à l'ordre collégial. Pendant la révision du programme collégial, en mars 1979, l'OIIQ, en collaboration avec la firme Sécor Inc., effectue une enquête auprès de ses membres afin de connaître leurs besoins et tracer un profil du membership en termes d'attitudes professionnelles. Les malaises exprimés furent nombreux et référaient à la perception professionnelle, aux activités à l'intérieur de l'équipe de soins et à la formation initiale (Côté, 1979: cité dans l'OIIQ, 1982). Une autre étude (Dulong, 1980: cité dans l'OIIQ, 1989), effectuée dans le cadre de l'évaluation du programme de formation des infirmières du niveau collégial, conclut que les infirmières ayant une formation collégiale ne répondent pas aux besoins du milieu, qu'elles semblent trop jeunes et n'ont pas la maturité nécessaire pour établir une relation d'aide, qu'elles manquent de planification et d'organisation, qu'elles intègrent mal leurs connaissances, qu'elles ont de la difficulté à appliquer la démarche $n u r s i n{ }^{4}$ en plus d'avoir des connaissances insuffisantes en déontologie.

Des recommandations sont faites concernant l'intégration de connaissances de base en sociologie, en politique, en administration, en relation d'aide, en pédagogie et enfin en gérontologie. L'acquisition de connaissances multiples et interdisciplinaires permettrait, selon la Direction générale des études collégiales (DGEC, 1987, p.75), «de rencontrer les objectifs d'une formation fondamentale en permettant l'articulation des apprentissages

4 Processus intellectuel composé de diverses étapes logiquement ordonnées ayant pour but le mieux-être du client (PHANEUF, M. (1986). Soins infirmiers, la démarche scientifique, Québec: McGraw-Hills Éditeurs, p.50). 
d'une discipline avec des apprentissages qui se rapportent directement à la pratique des soins infirmiers».

Quinze ans après le dépôt du rapport du Comité Rodger, en 1981, la FIIQ signale qu'il y a toujours une duplication au niveau de la formation de base de l'infirmière. En effet, deux programmes coexistent, encore aujourd'hui, l'un universitaire et l'autre de niveau collégial.

En 1982, lors de son Assemblée générale annuelle, l'OIIQ adopte une résolution mettant de l'avant que l'accès à l'exercice de la profession en l'an 2000 exigera une formation initiale de premier cycle universitaire en sciences infirmières. Pelland (1991, p.5) rappelle que depuis 1946, nous sommes toujours au même niveau de formation «[...] depuis 1946, où la formation des infirmières passait de deux années d'études après la neuvième année à trois années d'études après la onzième année, nous en sommes toujours au même point, au même niveau de formation». Comme le rapportent Riopelle et LeducLalonde (1982), la durée de la formation infirmière est de trois ans depuis le début du $\mathrm{XIX}^{\mathrm{e}}$ siècle. À ce jour, la formation de base de l'infirmière n'a jamais excédé une période de trois ans, indépendamment de l'ordre d'enseignement qui l'a assurée. Dans un même ordre d'idées, Walter (1988) précise l'importance d'un ancrage solide dans la pratique et dans la réalité de la vie, comme préalable à une formation de base de haut niveau professionnel afin que les élèves puissent saisir toute l'ampleur du savoir infirmier. Malgré la résolution de l'OIIQ qui exige une formation initiale universitaire, c'est dans la même année qu'est implanté dans les collèges de la province le nouveau programme Soins infirmiers 180.01 . 
Ainsi, dès le début des années 80 , quatre changements majeurs marquent la réforme du programme collégial en soins infirmiers: 1) la délimitation du rôle de l'infirmière diplômée des cégeps, 2) l'adoption d'un cadre théorique et d'une structure de cours, 3) une orientation systémique plutôt qu'une approche-contenu et 4) la transformation des programmes en programmes locaux (Goulet, O., 1993). Puisque le programme collégial en soins infirmiers issu de la réforme des années 80 est celui qui est enseigné actuellement dans les cégeps de la province, il nous semble important de situer chacun de ces changements pour bien comprendre la polémique actuelle autour de la formation en soins infirmiers. Pour expliquer la délimitation du rôle de l'infirmière diplômée des cégeps, Lambert (1993) précise que les personnes-ressources se sont inspirées de la conception de Marta Rogers. Selon Rogers (1975: cité dans Lambert, 1993), il doit y avoir deux voies de formation en soins infirmiers, l'une de niveau collégial et l'autre de niveau universitaire, pour satisfaire les besoins de la société. Toutefois, elle juge que les différences doivent être tangibles dans les savoirs et non dans les fonctions. Ainsi, selon les orientations de 1981, l'infirmière diplômée du collégial est qualifiée de généraliste. Le programme reconnaît les limites de la formation collégiale et précise que l'infirmière peut parfaire sa formation dans une université pour élargir son champ de compétence (Lambert, 1993). Concernant l'adoption d'un cadre théorique et d'une structure de cours, le programme d'études de 1981 comporte deux trames: l'une verticale qui représente le déroulement du programme dans le temps (organisation des sessions) et l'autre horizontale qui comporte cinq fils conducteurs qui découlent du cadre théorique. Ces fils sont la connaissance de la personne, la démarche de soins, la communication, l'engagement professionnel et l'actualisation de soi. Le cadre théorique, précise Lambert (1993), comporte trois niveaux: le premier détermine le but et le contexte de la pratique des soins infirmiers, le deuxième cerne la vision et les compétences requises pour la pratique des soins infirmiers et, le troisième établit les paramètres de la formation collégiale en soins infirmiers. Les caractéristiques du 
programme Soins infirmiers 180.01 devait permettre l'articulation des disciplines choisies pour servir de fondement aux soins infirmiers (DGEC, 1987). Le programme permet la transformation des plans-cadres en programmes locaux dans une perspective d'approcheprogramme. Toutes les personnes concernées par la formation devaient participer à la révision du programme d'études. Enfin, ce qui touche les changements qui modifient le visage de la formation collégiale en soins infirmiers, le nom du programme a été modifié afin de mieux refléter les nouvelles orientations du programme d'études. On ne parle plus de Techniques infirmières mais bien de Soins infirmiers pour le programme collégial. De plus, le temps alloué à la formation clinique est augmenté et les cours de biologie sont restructurés en fonction d'une pédagogie systémique.

Malgré ces changements majeurs apportés au programme collégial en soins infirmiers, la formation des infirmières demeure au cœur d'une controverse. C'est dans les années 90 que le dossier de la formation infirmière subit le plus de turbulences. En effet, ces années, caractérisées par des changements majeurs dans le domaine de la santé, ramènent plus intensément que jamais le dossier de la formation infirmière. Nous présentons quelques moments importants des dernières années, moments qui alimentent les discussions actuelles autour de la formation infirmière.

À l'automne 1995, la Direction des programmes entreprenait la réalisation d'une étude préliminaire dans le domaine des soins infirmiers. Les 6 et 7 mai 1996 ont lieu à Québec les États généraux de la profession infirmière. Les infirmières québécoises sont amenées à réfléchir sur l'avenir de leur profession. Plusieurs thèmes ont été discutés dans différents forums, mais tous les participants ont abordé le thème de la formation professionnelle et plusieurs mémoires sur le sujet ont été déposés. La lecture des Actes des États généraux de la profession d'infirmière (OIIQ, 1996) nous permet de constater qu'il 
n'y a pas eu d'entente sur la formation initiale de l'infirmière bien que certains points aient fait l'objet d'un consensus. Nous en rapportons quelques-uns :

- la formation doit être envisagée dans une perspective d'avenir;

- les habiletés et les compétences requises pour l'exercice de la profession d'infirmière doivent être définies;

- la formation doit intégrer les aspects socio-démographiques et préparer l'infirmière au travail;

- la formation doit être accessible dans toutes les régions du Québec;

- la diversité des niveaux de formation crée de la confusion en ce qui a trait à la reconnaissance de la profession d'infirmière;

- la concertation, dans une perspective de solidarité, est indispensable à la définition d'une formation de base et d'une formation continue;

- il faudrait prévoir un internat et explorer la possibilité d'une rémunération pour l'infirmière interne;

- la formation est un sujet de préoccupation et de division parmi les infirmières;

- la formation doit être axée davantage sur le rôle de l'infirmière (p.110-111).

Dans le cadre des États généraux de l'éducation, la problématique de formation infirmière a aussi été abordée. En effet, du 3 au 6 septembre 1996 avaient lieu à Montréal les assises nationales des États généraux de l'éducation dont le but était de faire un pas de plus vers la précision d'un projet de réforme de l'éducation et, de dégager les grands axes à retenir pour développer le futur projet éducatif. En septembre de la même année, l'Étude préliminaire sur les soins infirmiers était déposée au Comité national des programmes d'études professionnelles et techniques.

En octobre 1996, trois ateliers d'analyse de situation de travail ont eu lieu : les ateliers pour l'infirmière auxiliaire ont eu lieu à Québec les 9,10 et 11 octobre; les ateliers pour l'infirmière technicienne ont eu lieu à Montréal les 23, 24 et 25 octobre; les ateliers de l'infirmière bachelière les 30,31 octobre et $1^{\text {er }}$ novembre à Montréal. Certains commentaires et suggestions concernant la formation ont été émis dans les rapports d'analyse 
de situation de travail de l'infirmière diplômée du collégial et de celle diplômée de l'université. Nous avons choisi de rapporter ceux qui nous semblaient être significatifs aux diplômés des deux ordres d'enseignement:

L'infirmière diplômée du collégial (Infirmière diplômée et infirmier diplômé du collégial, Rapport d'analyse de situation de travail, 11 novembre 1996, p.51):

La formation devrait être axée sur le développement du savoir être et l'apprentissage de la relation d'aide. Les futures infirmières et les futurs infirmiers devraient posséder des habiletés en gestion.

L'utilisation d'équipements spécialisés en lien avec certains champs cliniques en particulier comme l'urgence, la psychiatrie et la salle d'opération ne devrait pas faire partie du programme d'études.

Les stages devraient être plus longs et mieux structurés.

Il est suggéré d'implanter une forme de jumelage entre les stagiaires et les infirmières et infirmiers en fonction.

L'infirmière diplômée de l'université (Infirmière diplômée et infirmier diplômé de l'université, Rapport d'analyse de situation de travail, 13 novembre 1996, p.51):

La formation en sciences infirmières semble varier beaucoup d'une université à l'autre, ce qui amène des différences considérables dans l'établissement de parallèles entre les études collégiales et universitaires.

Selon certaines personnes, l'infirmière formée au collégial serait davantage une exécutante tandis que l'infirmière formée à l'université serait une intervenante. Le programme d'études techniques devrait donc être orienté principalement vers l'exécution des soins tandis que le programme universitaire porterait davantage sur des aspects tels que la coordination, la gestion des soins, la recherche, l'évaluation et l'animation.

D'autres croient qu'il devrait exister une formation unique menant à la profession d'infirmière ou d'infirmier et que cette formation devrait être de niveau universitaire, ce qui permettrait une plus grande reconnaissance de la profession. 
Toujours en novembre 1996, le Conseil provincial de la formation infirmière était créé dans le but de soutenir l'harmonisation des programmes d'enseignement collégial et universitaire. Les membres du comité, au nombre de six, étaient deux représentants de l'OIIQ, deux représentants de l'enseignement collégial et deux représentants de l'enseignement universitaire (OIIQ, 1997). C'est également en novembre 1996 que la Direction générale de la formation professionnelle et technique du ministère de l'Éducation amorçait la révision du programme d'études techniques menant à la profession d'infirmière et d'infirmier. La révision du programme se fera selon l'approche par compétences tel que le prévoit le Renouveau de l'enseignement collégial (ministère de l'Éducation; sans date).

Le 20 janvier 1997, un Projet de formation collégiale en soins infirmiers était déposé dans un but de validation. Ce nouveau programme a été élaboré avec l'objectif de répondre à l'offre future d'infirmières au Québec. Une étude effectuée et publiée par l'AIIC (Association des infirmières et infirmiers du Canada) en octobre 1997, estimait l'offre future d'infirmières autorisées au Canada et précisait le niveau de formation. Cette étude précise que les connaissances requises pour la pratique infirmière de l'avenir devront être de niveau universitaire afin de répondre aux soins de plus en plus complexes du milieu. Enfin, une autre étude effectuée récemment par le groupe Sécor (1996) rapporte que la réforme de la santé exige de nouvelles habiletés et attitudes de la part des professionnels de la santé, lesquelles habiletés et attitudes doivent être développées pendant la formation:

- acquérir des compétences en matière d'autonomie professionnelle, de leadership et de gestion;

- comprendre et appliquer de la technologie de complexité grandissante et ce, autant pour les soins livrés en milieu hospitalier qu'à domicile;

- être capables de travailler en collaboration avec les autres professionnels pour intégrer une variété de services et d'activités visant à promouvoir et améliorer la santé; 
- participer aux efforts pour assurer des soins de qualité aux individus, aux familles et aux communautés, et une utilisation plus efficace des ressources;

- impliquer les individus et les familles dans les décisions concernant leur santé et les soins;

- gérer un large volume d'information scientifique et technologique et être à même d'utiliser l'informatique;

- participer à des discussions au sein des équipes de soins sur les questions d'ordre éthique et être en mesure de conseiller les individus et les familles confrontés à de telles décisions;

- anticiper les changements dans le système de santé et répondre en assurant par un processus continu, la mise à jour de leurs connaissances afin de développer leurs compétences cliniques et leur autonomie professionnelle (p.27-28).

En janvier 1997, le ministre de la Santé et des Services sociaux confiait à un groupe de travail sur les soins infirmiers (le Comité Rochon) le mandat d'analyser les impacts de la transformation du réseau sur les besoins en soins infirmiers et d'assistance, dans trois grandes missions d'établissements du réseau à savoir les CHSGS (Centre hospitalier de soins généraux et spécialisés), les CHSLD (Centre hospitalier de soins de longue durée) et les CLSC (Centre local de services communautaires). Le rapport final de ce Comité a été présenté en novembre 1997. Ce comité avait pour mandat de proposer au Ministère de la Santé et des Services sociaux des orientations concernant la main-d'œuvre en soins infirmiers dans le contexte de transformation du réseau de la santé, de l'évolution des besoins et des attentes de la population en matière de soins de santé, de l'évolution de la pratique médicale, de l'évolution des sciences infirmières, de l'évolution des connaissances et de la technologie, du contexte prévisible des finances publiques et de la nécessaire recherche de la plus grande efficience possible (Groupe de travail sur les soins infirmiers, 1997). Le Comité a proposé au Ministère quatre scénarios différents qui ont permis d'alimenter les discussions et envisager les implications potentielles pour la profession infirmière. Nous occultons volontairement du texte les scénarios 1,2 et 3 et rapportons uni- 
quement le «scénario 4», scénario sur lequel le groupe de travail a porté son choix (Groupe de travail, 1997, p.13):

[...] le scénario 4 favoriserait une révision en profondeur du système de formation selon une conception de l'organisation du travail en soins infirmiers axée sur deux types de ressources polyvalentes, soit une ressource orientée vers l'assistance aux personnes dont la formation serait de niveau secondaire et axée sur les besoins de la vie courante et les soins d'hygiène d'une part, et une ressource dont la formation serait donnée au CÉGEP et axée sur les techniques infirmières. Pour certains champs d'expertise identifiés, cette formation pourrait être complétée en milieu universitaire.

Suite au dépôt du rapport du comité Rochon, en mai 2000, le ministre de l'Éduca-

tion, Monsieur François Legault, informait les cégeps des orientations:

[...] les recommandations qui sont formulées dans ce rapport ainsi que les travaux menés par le ministère de la Santé et des Services sociaux (MSSS) au sein du Forum national de planification de la main-d'œuvre des infirmières et des infirmiers nous fournissent des constats importants. Ils nous démontrent que les exigences de formation sont de plus en plus grandes en raison des modifications apportées à la pratique infirmière et de la transformation récente du réseau de la santé. Ils nous informent également que le réseau de la santé requerra un très grand nombre d'infirmières pour les quinze prochaines années. Les recommandations du Comitéconseil ont fourni des éléments incontournables dans le choix des orientations suivantes. Ainsi, il me paraît important de maintenir une filière de formation initiale qui permette à toute personne titulaire d'un diplôme d'études collégiales (DEC) en formation préuniversitaire ( 2 ans) d'accéder au programme universitaire en sciences infirmières ( 3 ans). De plus, l'élaboration d'un continuum de formation sur cinq années, soit la formation technique en soins infirmiers suivie d'une formation universitaire en sciences infirmières, est également retenue. Ce continuum permettra l'accès au permis d'exercice de l'Ordre des infirmières et infirmiers du Québec (OIIQ) après l'obtention du DEC et il facilitera la poursuite à l'université de la formation infirmière. Afin de mettre en œuvre cette orientation, un comité de travail est mis en place. Il sera composé de représentantes et de représentants des collèges, des universités et de l'OIIQ. Mme Pauline ChampouxLesage, sous-ministre de l'Éducation, en assumera la présidence. Le sous-ministre adjoint à la Direction générale des politiques de main-d'œuvre du ministère de la Santé et des Services sociaux (MSSS) sera invité à participer au comité à titre d'observateur. Le mandat de ce comité, au cours des six prochains mois, sera d'établir le cadre d'intégration des programmes d'études techniques et universitaires concernés. 
Il est prévu que le programme d'études collégiales révisé Soins infirmiers soit implanté dès le trimestre d'automne 2001 (Lettre de deux pages adressée aux Directeurs et Directrices des études des cégeps de la province de Québec, le 30 mai 2000, par le ministre d'État à l'Éducation et à la jeunesse, Monsieur François Legault).

Ainsi, dès l'automne 2001, le nouveau programme Soins infirmiers 180.A0, orienté par l'approche par compétences, sera implanté dans les collèges de la province.

C'est dans le cadre de ce nouveau programme collégial en soins infirmiers que notre question générale de recherche prend place. Rappelons-la donc: la formation collégiale en soins infirmiers peut-elle répondre aux nouvelles demandes sociales?

Dans un premier temps, afin d'identifier les nouvelles orientations qui commandent des changements dans la pratique infirmière, nous procéderons à une analyse de contenu à partir de documents publiés par des organismes qui s'intéressent à la formation infirmière soient le MEQ, l'OIIQ, la FIIQ et l'ADRSI.

Par la suite, nous examinerons le projet du programme Soins infirmiers 180.A0 pour voir dans quelle mesure il pourrait répondre aux demandes sociales actuelles en soins de santé.

Le chapitre suivant, réservé à la méthodologie, nous permettra de contextualiser les textes publiés par les instances précitées et de justifier notre démarche d'analyse. 


\title{
CHAPITRE III
}

\author{
Méthodologie
}

\subsection{Introduction}

Étant de nature qualitative, cette étude s'appuie sur un ensemble des principes et des caractéristiques propres à ce genre de recherches. Dans notre recherche, nous procéderons à l'analyse de plusieurs textes, à la recherche de sens dans le but de mieux comprendre le phénomène social que nous étudions, c'est-à-dire la formation en soins infirmiers en lien avec les nouvelles demandes sociales du réseau de la santé.

L'accès à des textes produits par différentes institutions, entre 1989 et 1996, et la similitude quant à l'objet discursif (la formation de l'infirmière), nous oriente vers l'analyse de contenu comme choix méthodologique. Effectivement, ces documents produits suite aux réformes de l'éducation et de la santé qu'a connues le Québec au début des années quatre-vingt-dix, constituent une banque de données très significative qui se prête bien à l'analyse. Mais avant de procéder à l'analyse et de présenter nos sources de données, nous souhaitons rapporter les propos de quelques auteurs sur les origines et l'intérêt de l'analyse de contenu comme méthode de collecte et d'analyse des données. 
C'est au cours des années 30 que l'analyse de contenu a vu le jour en sciences sociales (Mayer et Ouellet, 1991). À l'origine, cet outil fut développé et utilisé par Lasswell et quelques autres chercheurs, pour étudier l'opinion publique (Mayer et Ouellet, 1991). Dans les années 50 aux États-Unis, il semblerait que les procédures de l'analyse de contenu aient été au centre d'un débat (George, 1959: voir Bardin, 1977). À cette époque, certains chercheurs défendaient la validité d'une analyse quantitative alors que d'autres affirmaient que le caractère qualitatif était ce qui définissait l'analyse de contenu (George, 1959: cité dans Bardin, 1977). George élabora un tableau comparatif de ces deux conceptions et précisa que les deux méthodes n'ont pas le même terrain:

L'approche quantitative est fondée sur la fréquence d'apparition de certains éléments du message. [...] obtient des données descriptives par une méthode statistique. Elle semble, grâce au décompte systématique, plus précise, plus objective, plus fiable et fidèle, car l'observation y est davantage contrôlée. Rigide cependant, elle est utile dans les phases de vérification des hypothèses (1959: cité dans Bardin, 1977, p.147-148).

L'approche qualitative correspond à une procédure plus intuitive mais aussi plus souple, plus adaptable à des indices non prévus ou à l'évolution des hypothèses. Elle permet de suggérer des relations possibles entre un indice du message et une ou plusieurs variables du locuteur. Elle est surtout valable pour faire des déductions spécifiques à propos d'un événement, d'une variable d'inférence précise et non pour des inférences générales. Elle peut fonctionner sur des corpus réduits et établir des catégories plus discriminantes puisqu'elle n'est pas liée comme l'analyse quantitative à des catégories donnant lieu à des fréquences suffisamment élevées pour que les calculs soient possibles (1959: cité dans Bardin, 1977, p.147148).

Si la méthode qualitative semble plus souple et laisse davantage parler le discours, elle comporte toutefois, selon George (1959: cité dans Bardin, 1977), certains inconvénients:

Elle pose des problèmes au niveau de la pertinence des indices retenus puisqu'elle sélectionne ces indices sans traiter exhaustivement tout le contenu. Il y a des risques de laisser des éléments importants ou de prendre en compte des éléments 
non significatifs. La compréhension exacte du sens est capitale dans ce cas. De plus, jouant sur des éléments isolés ou des fréquences faibles, elle voit s'accroître le risque d'erreur. D'où l'importance du contexte. Contexte du message mais aussi contexte extérieur à celui-ci: quelles sont les conditions de production, autrement dit, qui parle à qui, dans quelles circonstances, quel est le moment et le lieu de la communication, quels sont les événements antérieurs ou parallèles (George, 1959: cité dans Bardin, 1977, p.147-148).

De plus, George (1959: cité dans Bardin, 1977, p.147-148) laisse entendre que les deux méthodes peuvent être utilisées parallèlement dans une même recherche: «Enfin, précisons que l'analyse qualitative ne rejette pas toute forme de quantification. Ce sont les indices qui sont retenus de manière non fréquentielle».

Cet auteur définit l'analyse de contenu comme:

[...] un ensemble de techniques d'analyse des communications visant, par des procédures systématiques et objectives de description du contenu des messages, à obtenir des indicateurs (quantitatifs ou non) permettant l'inférence de connaissances relatives aux conditions de production/réception (variables inférées) de ces messages (Bardin, 1977, p.47).

L'objet de l'analyse de contenu, selon Bardin (1977), est l'aspect individuel du langage, c'est-à-dire la parole, et vise la connaissance de certaines variables (psychologiques, sociologiques, etc.) à partir d'un échantillon de messages particuliers. «Elle prend en compte les significations (contenu) et éventuellement leur forme et la distribution de ces contenus et formes» et elle «cherche à savoir ce qui est derrière les paroles sur lesquelles elle se penche» (p.48). «Elle est un ensemble de techniques d'analyse des communications» utilisant plusieurs outils différents tout dépendant de son champ d'application (p.35). 
L'auteur insiste sur l'importance de distinguer l'analyse de contenu de la linguistique et de la lexicologie et spécifie que cette distinction se situe principalement au niveau de l'objet d'étude. «L'objet de la linguistique est la langue, c'est-à-dire l'aspect collectif et virtuel du langage, celui de l'analyse de contenu est la parole, c'est-à-dire l'aspect individuel et actuel (acte) du langage» (Bardin 1977, p.48). L'analyse de contenu vise la connaissance de certaines variables et se rapproche davantage de la lexicologie qui est l'étude du vocabulaire parce que les deux méthodes ont recours à un système de classification et fonctionnent sur des unités de signification, c'est-à-dire le mot.

Aujourd'hui, Kelly (1984: cité dans Mayer et Ouellet, 1991) rapporte que la grande majorité des recherches empiriques dans lesquelles on utilise l'analyse de contenu, se rattachent principalement à la sociologie-anthropologie, aux sciences de la communication et aux sciences politiques. Le même auteur ajoute que la popularité grandissante de cette méthode provient de l'attention de plus en plus importante que l'on porte à l'analyse de la communication humaine (1984: cité dans Mayer et Ouellet, 1991). Pour cet auteur, il s'agit d'étudier les communications en se servant d'une stratégie de vérification ayant comme objectif de répondre à la question suivante: qui dit quoi, à qui, comment et avec quel effet? De plus, selon Rongère (1979: cité dans Mayer et Ouellet, 1991; L'Écuyer, 1991), il existe plusieurs types d'analyse de contenu et que chacun des types est approprié à une ou plusieurs sciences sociales: l'analyse historique, l'analyse juridique, l'analyse littéraire, l'analyse linguistique, l'analyse psychologique et l'analyse sociologique ou psychosociologique.

Pour être efficace, l'analyse de contenu doit répondre à certaines exigences, entre autres, à l'utilisation de règles rigoureuses pour atteindre les buts visés et la mise au point d'un système de classification rigoureux respectant un certain nombre d'étapes précises 
(L'Écuyer, 1991, p.31-32). Pour Bardin (1977) et L'Écuyer (1991), la classification ou «catégorisation» constitue une étape importante de la démarche dans l'analyse de contenu car elle est l'étape où le chercheur doit organiser le matériel par catégories. Bardin (1977) rapporte que les différentes phases de l'analyse de contenu doivent s'organiser autour de trois pôles:

1) La préanalyse ou phase d'organisation où le chercheur en plus de choisir les documents à soumettre à l'analyse, formule des hypothèses et des objectifs et élabore des indicateurs qui lui permettront d'interpréter les résultats.

2) L'exploitation du matériel, étape la plus longue de l'analyse, consiste en des opérations de codage en fonctions des consignes formulées. C'est-à-dire qu'il s'agit de l'étape où le chercheur transformera le matériel pour en arriver à se représenter le contenu.

3) Le traitement des résultats, l'inférence et l'interprétation; étape où les résultats seront traités de manière à être significatifs et valides.

Cette brève recension des écrits sur l'analyse de contenu nous permet de situer notre recherche dans un cadre social bien précis. En effet, le cadre social qui nous intéresse plus spécifiquement se situe entre les années 1989 et 1997, moment où la profession infirmière a fait l'objet de deux réformes, soit celle de l'éducation et celle de la santé. Comme il a déjà été mentionné dans le chapitre précédent, la formation en soins infirmiers soulève de nombreuses interrogations depuis plusieurs années mais nous limitons notre analyse à des textes produits suite à des événements majeurs qui ont eu lieu dans les domaines de l'éducation et de la santé.

Dans la présente recherche, nous choisissons d'utiliser la méthode qualitative car le contexte de production des discours à soumettre à l'analyse nous parait très significatif. De plus, nous voulons extraire des discours les éléments importants et chercher des relations entre les différents discours qui ont été produits dans un contexte spécifique. 
Afin de respecter la phase d'organisation, nous allons présenter les organismes qui s'intéressent à la formation infirmière en plus des textes qui ont servi à la constitution de notre corpus.

\subsection{Constitution du corpus}

L'analyse de contenu se fait à partir de documents publiés entre les années 1989 et 1997, période pendant laquelle la profession infirmière a été influencée par deux réformes, c'est-à-dire celle de l'éducation et celle de la santé. Ces documents ont été publiés par les différents organismes qui s'intéressent de près ou de loin à la formation des infirmières, c'est-à-dire le ministère de l'Éducation du Québec, l'Ordre des infirmiers et infirmières du Québec, la Fédération des infirmières et infirmiers du Québec et l'Association des directeurs et responsables de soins infirmiers du Québec. Comme nous l'avons déjà mentionné, la formation infirmière a fait couler beaucoup d'encre depuis quelques années et l'abondance des documents produits nous oblige à restreindre notre corpus. Dans le cadre de cette recherche, nous limitons notre analyse aux ouvrages suivants:

(1996) Soins infirmiers. Étude préliminaire. Direction générale de la formation professionnelle et technique, Direction des programmes, 180 pages.

(1996) Les effectifs de la profession infirmière: analyse prospective et enjeux. Document préparé par le groupe Sécor Inc. pour l'OIIQ, 70 pages.

(1995) Mémoire déposé dans le cadre de la Commission des États généraux sur l'Éducation. La Fédération des Infirmières et Infirmiers du Québec, 53 pages.

(1993) L'évolution des soins infirmiers au Québec: pour une dynamique d'amélioration continue. La réflexion de l'Association des directeurs et responsables des soins infirmiers du Québec, 27 pages. 
(1989) Projet 2000. Résumé. Le baccalauréat pour l'admission à la profession d'infirmière ou d'infirmier en vue de la santé pour tous. Ordre des infirmières et infirmiers du Québec, 69 pages.

Ces textes, qui ont servi à construire notre corpus, ont été pour la plupart publiés à la suite des événements suivants:

- la réforme de la formation infirmière 1982;

- la nouvelle réforme de la santé et des services sociaux implantée au début des années 90;

- la Journée d'étude de l'ADRSI tenue le 15 janvier 1993;

- les États généraux de l'éducation tenus à travers la province de Québec en 1995;

- les États généraux de la profession infirmière qui se sont tenus à Québec les 6 et 7 mai 1996.

Les textes retenus pour fins d'analyse proviennent de documents officiels, c'est-àdire qu'ils ont tous été élaborés et diffusés par des organismes publics concernés par la formation et la pratique infirmières:

l'OIIQ (Ordre professionnel des infirmières et infirmiers du Québec); la FIIQ (Fédération des infirmiers et infirmières du Québec); l'ADRSI (Association des directeurs et responsables des soins infirmiers); le MEQ (ministère de l'Éducation du Québec).

Nous présentons une brève description du rôle spécifique de chaque organisme:

L'Ordre professionnel des infirmières et infirmiers de la province du Québec (OIIQ) est une corporation professionnelle qui regroupe 67,000 membres à travers le Québec. Sa mission, confiée par le législateur via le Code des professions, est d'assurer la protection du public par le contrôle et l'évaluation de la pratique infirmière. 
La Fédération des infirmiers et infirmières du Québec (FIIQ) s'engage sur le terrain des luttes syndicales, sociales et économiques à promouvoir une société qui soit à la recherche d'une justice, d'une répartition équitable des richesses et des pouvoirs et ce, sans affiliation à un parti politique. La FIIQ assure la représentation syndicale d'un grand nombre d'infirmiers et d'infirmières à travers la province en assurant le respect des droits de celles-ci (FIIQ; http://www.fiig.qc.ca).

Le ministère de l'Éducation du Québec (MEQ), instance centrale en matière éducative dans la province, évalue et élabore les programmes d'enseignement, incluant ceux de niveau collégial, dont celui en soins infirmiers.

L'Association des directeurs et responsables des soins infirmiers du Québec (ADRSI) regroupe les directrices et responsables de toutes les catégories d'établissements qui dispensent des soins infirmiers afin de permettre la représentativité globale de tous les aspects de la gestion des services de soins infirmiers au Québec. Le conseil d'administration de l'ADRSI a tenu, le 15 janvier 1993, une journée d'études sur l'avenir des soins infirmiers au Québec et les orientations à privilégier pour les prochaines années.

Nous pensons qu'une analyse de contenu, à partir des textes produits par ces organismes, peut nous aider à dégager certaines tendances partagées par les différents organismes ainsi qu'à cerner les représentations que ces organismes ont de la formation infirmière en relation aux demandes sociales actuelles en soins infirmiers. Comme nous l'avons mentionné antérieurement, l'analyse de contenu comporte plusieurs étapes d'élaboration. Nous précisons, dans les paragraphes suivants, l'organisation de notre recherche en rapport avec ces différentes étapes. 
Plusieurs lectures des documents du corpus constituent notre démarche d'analyse. Une première lecture nous permet de repérer certains indices, de trouver des idées ainsi que de formuler nos premières hypothèses interprétatives. Au terme de cette première lecture, nous observons que les différents discours utilisent un langage similaire; on peut affirmer que les paroles des discours présentent certaines similitudes.

Une deuxième lecture nous permet de mettre en relief ces similitudes dans les discours et de classer le matériel d'analyse selon les trois grands thèmes auxquels semblait s'intéresser l'ensemble des organismes: le contexte social, le contexte professionnel et le contexte de formation. Par la suite, nous classons, pour chacun des discours, les éléments se rapportant à chaque thème. Tous les discours sont découpés selon les trois thèmes que nous avons cités précédemment. Par la suite, nous mettons en évidence les points spécifiques de chaque discours, pour chacun des thèmes, pour faire ressortir, à partir d'une lecture comparative, les points saillants. Ce sont ces points saillants qui nous permettent de mettre en évidence la position des différents organismes par rapport à la formation infirmière dans le contexte des nouvelles demandes sociales.

Cette lecture analytique nous permet d'atteindre notre premier objectif, c'est-à-dire d'analyser les nouvelles orientations de la pratique infirmière. De plus, comme l'implantation d'un nouveau programme collégial en soins infirmiers est prévue pour l'automne 2001, nous examinons dans quelle mesure il saura répondre aux nouvelles demandes sociales. Le nouveau programme Soins infirmiers 180.A0 étant élaboré selon l'approche par compétences, nous utilisons un document de travail publié par le ministère de l'Éducation en décembre 2000 intitulé Projet de programme d'études en soins infirmiers pour procéder à cette partie de l'analyse. 
Toutefois, avant de procéder à l'étude de ce nouveau programme et rapporter quelques notions sur l'approche par compétences, il nous apparaît essentiel de revenir à l'analyse de contenu. Le chapitre qui suit présente une analyse de contenu divisée en trois grands thèmes (contexte social, contexte professionnel et contexte de formation) à partir des textes que nous avons annoncés précédemment. Nous tentons d'aller au-delà de la parole en repérant certains indicateurs nous permettant de savoir ce qui se cache derrière la parole explicite. 


\section{CHAPITRE IV}

\section{Présentation et analyse de contenu}

Ce chapitre présente les résultats de l'analyse de contenu à laquelle ont été soumis les documents qui constituent notre corpus. Ce chapitre comprend trois sections qui décrivent et analysent successivement les trois contextes qui nous permettent de comprendre les transformations sociales et les exigences auxquelles la profession infirmière est confrontée.

\subsection{Contexte social}

L'analyse nous permet de constater que tous les organismes sont unanimes pour dire que le réseau de la santé du Québec s'oriente vers une nouvelle philosophie. Les facteurs à la base de ce changement font suite à des rencontres internationales et nationales et à la difficulté financière de l'État à répondre aux besoins croissants des citoyens. Autant le ministère de l'Éducation que l'Ordre des infirmières et infirmiers du Québec y font allusion dans leurs discours respectifs.

La transformation du système sociosanitaire québécois dans les années 90 est issue de la réorientation des politiques gouvernementales de la province en matière de santé et de bien-être des populations, sous l'influence de deux facteurs: la redéfinition, sur les plans international et national, du concept santé et de bien-être, et la crise des finances publiques (MEQ, 1996, p.9). 
La crise actuelle des systèmes de santé dans le monde est provoquée par deux grands types de pressions: une demande accrue pour des soins de santé d'une part, et l'incapacité des «payeurs» de soutenir le rythme rapide de croissance des coûts (OIIQ, 1996, p.5).

Du paradigme curatif, qui qualifiait jusqu'à tout récemment les soins de santé, nous nous dirigeons vers un paradigme de promotion et de prévention de la santé. Dorénavant, on parle de paradigme de santé, de bien-être, de santé pour tous et d'approche globale de la santé:

D'abord axé sur des soins hospitaliers curatifs, le système s'oriente vers la promotion de la santé et du bien-être des individus, des familles et des communautés dans leur milieu de vie (OIIQ, 1989, p.v).

Le paradigme gestion de la santé s'est imposé comme supérieur à celui de la gestion des soins parce qu'il replaçait au centre du système, la santé de l'individu, parce que l'approche préventive s'imposait comme plus efficace et moins coûteuse que l'approche curative, parce qu'avec le principe de la capitation, les payeurs se transformaient en acheteurs, se mettant alors en position d'exercer un pouvoir économique considérable et d'influencer le système de livraison de soins. Ce concept est en train de donner lieu à une mutation sans précédent de l'industrie des soins de santé aux États-Unis, et d'influencer les systèmes européens et canadiens (OIIQ, 1996, p.16).

Dans le même sens, le ministère de l'Éducation fait siens les propos du ministère de Santé et Services Sociaux :

Considérant que «la santé et le bien-être entretiennent des liens très étroits, qu'ils représentent en quelque sorte les deux faces d'une seule et même réalité», tenant compte par ailleurs de l'influence des facteurs économiques, sociaux et culturels sur la santé et le bien-être, le MSSS redéfinit, dans cette Loi, ces deux derniers concepts, non plus comme l'absence de maladie et de problèmes sociaux mais comme la capacité physique, psychique et sociale d'une personne d'agir dans son milieu et d'accomplir les rôles qu'elle entend assumer, d'une manière acceptable pour ellemême et pour les groupes dont elle fait partie (MEQ, 1995, p.11). 
Cette nouvelle philosophie de la santé, pour l'ensemble des organismes consultés, place le citoyen au cœur de l'action et le responsabilise face à la prise en charge de sa propre santé. On désire que le citoyen s'informe et qu'il adopte des comportements lui permettant de se maintenir en santé; on l'encourage à s'impliquer davantage dans la prise en charge de sa santé: «On réaffirme, en outre, l'individu, les familles et les milieux de vie sont les premiers acteurs de leur propre santé et de leur bien-être» (MEQ, 1995, p.11).

L'Ordre fait référence à ces acteurs en terme de partenariat, ce qui lui attribue une responsabilité face à leur santé à partager avec les services de santé. Elle dit :

L'usager deviendra donc un partenaire à part entière qui devra être informé sur sa situation de santé, sur les choix à faire, sur les coûts des soins ainsi que sur les résultats attendus. L'usager sera responsable de sa santé et prendra les décisions après avoir été bien informé (OIIQ, 1996, p.42).

Pour favoriser l'atteinte des objectifs de la nouvelle politique de santé, les organismes s'entendent pour dire que la façon de distribuer les soins devra être revue. Une réorganisation du réseau, où les services offerts seront plus près de la communauté, permettra de mieux rejoindre les citoyens. L'OIIQ voit dans l'acquisition des compétences par les infirmières un facteur de réussite de la nouvelle organisation des soins de santé, tandis que le MEQ met l'accent sur l'accessibilité des services et reconnaît qu'une analyse des programmes de formation s'impose:

La réorganisation des soins de santé est une nécessité. Pour parvenir avec succès au terme de la réforme, de profonds changements, structurels et culturels, devront se produire. Les individus impliqués dans la livraison des soins devront également faire des efforts importants pour acquérir les compétences et habiletés nécessaires à la réussite de la transformation en cours (OIIQ, 1996, p.28).

Fondamentalement, il s'agit de réorienter l'organisation et le fonctionnement du réseau de la santé et des services sociaux, afin d'offrir «les bons services au bon en- 
droit, et au bon moment» et d'assurer la plus grande accessibilité possible aux services tout en ayant recours aux «solutions les plus simples et les plus efficaces» et en comptant sur «l'implication étroite des personnes, des groupes et des communautés dans les interventions qui leur sont destinées»(MEQ, 1996, p.11). D'autre part, la pertinence et la cohérence des programmes de formation actuellement offerts doivent être analysés au regard des bouleversements du marché du travail (MEQ, 1996, p.1).

Nous avons vu que ces transformations donnent lieu à un recentrage des missions, au regroupement, à la fermeture ou à la fusion de certains établissements pour une meilleure efficacité et une plus grande efficience. Parallèlement, on tend à développer de nouveaux services tels que les centres ambulatoires dans les hôpitaux, les cliniques spécialisées, le service Info-santé et les hôpitaux de jour. Ces transformations sont par ailleurs conduites dans une perspective de soins et de services intégrés (MEQ, 1996, p.25).

Cette réorganisation du système de santé, selon les organismes, est essentielle pour répondre aux nouveaux besoins des usagers. Ces nouveaux besoins émergent des changements multiples au sein de la société et sont d'ordres variés. Les principaux changements rapportés par les organismes font référence au vieillissement de la population, à la pluralité ethnique, aux modifications du tissu socio-géographique, ainsi qu'aux attentes de la population:

Parmi ces changements, nous pouvons déjà observer quelques tendances importantes comme le vieillissement de la population, la pluralité ethnique, l'apparition de nouvelles maladies ou la résurgence de certaines autres, le développement rapide des techniques de diffusion de l'information, l'essor des médecines alternatives et finalement l'impact du phénomène de société tels que le chômage, la pauvreté et l'éclatement familial (FIIQ, 1995, p.19).

En outre, de nouvelles réalités sociales font naître de nouveaux besoins de santé. Pensons à la diversité ethnique de la population, à la détérioration des conditions générales de vie, des femmes monoparentales et des jeunes de milieux défavorisés, à l'apparition de nouvelles maladies comme le sida, ou encore, à l'incidence de la drogue sur la santé et l'autonomie fonctionnelle et sociale des usagères et des usagers (MEQ, 1996, p.4). 
Non seulement les besoins de la population changent mais ses attentes se modifient. Les patientes et les patients sont devenus des "consommateurs» de soins de santé, des «usagers» du système de santé. Depuis le début des années 70 , avec la mise en place de regroupement de bénéficiaires, les usagères et les usagers du système de santé entendent être informés de toute question relative à leur état de santé, être respectés en tant qu'individus, voire même participer aux prises de décision concernant leur santé (MEQ, 1996, p.45).

Pour répondre à ces nouveaux besoins, les réseaux public et communautaire, comme le rapportent les documents analysés, devront travailler en étroite collaboration:

La réforme du réseau de la santé et des services sociaux fait la promotion active de la coordination inter-établissements (ADRSI, 1993, p.15).

Il est donc à prévoir que la place des organismes communautaires en matière de santé pourrait aller en s'accroissant au cours des prochaines années, soit par la multiplication de ces organismes, soit par la croissance de ceux qui existent déjà (MEQ, 1996, p.23).

Les deux secteurs qui pourraient connaître le plus important développement semblent être celui du maintien à domicile, vu le mouvement tendant à réduire les séjours dans les hôpitaux et les institutions d'hébergement, et celui de la santé mentale (MEQ, 1996, p.23).

L'accent sera donc mis sur une utilisation optimale des ressources des centres hospitaliers, un retour rapide à domicile et la mise en place dans la communauté d'un réseau de support intégré (OIIQ, 1996, p.44).

L'analyse nous révèle également, qu'au-delà de la réorganisation des soins de santé et de l'émergence des nouveaux besoins des usagers, c'est la transformation du rôle de l'infirmière qui permettra de faciliter l'intégration de cette nouvelle philosophie de la santé au sein de la communauté. Si pour l'OIIQ il s'agit d'un élargissement intéressant du rôle de l'infirmière dans la gestion des soins, pour la FIIQ, il ressort que l'évolution des soins infirmiers, dans le contexte de la réforme, contribuera à augmenter les pressions que subissent déjà les infirmières dans leur milieu de travail: 
Le centre de gravité du nouveau système de santé reposera sur les intervenants de première ligne qui auront la responsabilité d'assurer le maintien de l'état de santé de populations spécifiques et de gérer les épisodes de soins (OIIQ, p.32, 1996).

Il ressort de la réalité démographique québécoise et de son évolution que les soins infirmiers subiront des pressions qui pourraient en certains cas en modifier la nature, la qualité et la quantité (FIIQ, p.21, 1995).

Pour le MEQ et l'ADRSI, la place qu'a pris l'évolution des technologies dans la société actuelle n'est pas étrangère au changement du rôle de l'infirmière dans les différents milieux de soins:

Bref, la transformation des besoins et des attentes de la population, l'évolution des technologies et les changements d'orientation dans la distribution des soins et des services semblent avoir des incidences importantes sur le travail du personnel en soins infirmiers, que ce travail soit effectué en établissement ou à domicile. Ils transforment les méthodes de travail, diversifient les procédés utilisés et multiplient les activités (MEQ, 1996, p.46).

Notre défi primordial est d'adapter les soins et services que nous dispensons aux nouvelles réalités: usagers, technologies, ressources, etc. Pour continuer de progresser dans ce contexte de mutations et de turbulences, nous devrons revoir en profondeur nos rôles, nos approches, nos modes d'organisation. Et pour être en mesure de réaliser ces transformations, nous aurons besoin d'infirmières dotées d'une formation plus avancée, dans un cadre professionnel révisé (ADRSI, 1993, p.22).

Lorsqu'on fait référence au rôle de l'infirmière, on touche la dimension "professionnelle» de sa pratique. Cette dimension fait référence aux deux composantes de l'exercice infirmier, soient les actes délégués qui se rapportent au traitement des ordonnances et les actes autonomes qui se rapportent au jugement clinique de l'infirmière. De la réorganisation du système et de la nouvelle philosophie qui le sous-tend, émerge un nouveau rôle de l'infirmière que nous allons identifier en présentant l'analyse du contexte professionnel. 


\subsection{Contexte professionnel}

Les modifications du réseau de la santé auront des répercussions majeures sur le travail des infirmières, comme nous le révèle l'analyse des discours des organismes étudiés. Plus particulièrement, ces changements auront des répercussions sur «l'aspect professionnel» de la pratique infirmière. En effet, l'analyse des discours officiels nous permet, dans un premier temps, d'affirmer que chaque organisme reconnaît l'apport de la profession infirmière au système de santé. Pour l'OIIQ, les infirmières sont les actrices principales des changements dans les soins car elles sont les leaders du système de santé.

L'Ordre fait siennes les déclarations de l'Organisation Mondiale de la Santé ainsi que celles du MSSS afin de qualifier le rôle primordial que les infirmières jouent au sein de la société. Elle affirme :

En 1978, l'OMS déclarait que les infirmières avaient la responsabilité de changer les soins (OIIQ, 1989, p.13).

[...] l'infirmière est la principale intervenante en santé auprès de la population. Le ministre de la Santé et des Services sociaux a d'ailleurs qualifié cette professionnelle «d'épine dorsale du système de santé» (OIIQ, 1989 p.11).

[...] on est à même de constater que les infirmières prennent des décisions qui affectent les initiatives personnelles des gens et qui peuvent les rendre capables d'exercer plus de contrôle, qu'ils ne le font maintenant, sur leur qualité de vie. Elles fournissent directement ou indirectement leur service par le contact privilégié qu'elles établissent avec la clientèle et les membres de l'équipe multidisciplinaire ou en cultivant des alliances avec des responsables d'autres secteurs que celui du sanitaire (OIIQ, 1989, p.11).

[...] la responsabilité du corps infirmier québécois est d'assumer constamment son leadership en "prenant soin» de la population à travers les phénomènes de la vie qui influencent l'état de santé des individus, des familles et de la communauté (OIIQ, 1996, p.2). 
Dans une perspective semblable, la FIIQ considère les infirmières comme des professionnelles au cœur du système de santé:

Les infirmières, de par leur formation, sont les professionnelles capables de relever les défis que pose la mutation graduelle de nos services de santé vers une approche plus préventive et basée sur une conception globale, multidisciplinaire et communautaire. Bien au-delà des techniques de soins, leurs intentions se sont toujours situées dans une approche globale, s'adressant aux personnes d'abord et non seulement à la maladie (FIIQ, 1995, p.27).

Dans tous les types d'établissements, l'infirmière est ainsi au cœur de la santé et des soins (FIIQ, 1995, p.30).

L'analyse nous permet de constater que les organismes accordent une très grande importance à la modification du rôle de l'infirmière dans le contexte de la réforme de la santé. Les changements actuels engendrés par une nouvelle philosophie de la santé amènent une transformation du rôle de l'infirmière. Dorénavant, l'infirmière sera appelée à travailler autrement pour répondre à de nouveaux besoins. Le MEQ est aussi sensible aux changements, que le rôle de l'infirmière est en train de souffrir. Selon cet organisme, le virage ambulatoire aura un impact considérable sur le travail des infirmières:

En relation avec les objectifs du virage ambulatoire et dans la perspective de la nécessaire concertation interdisciplinaire, une nouvelle modalité de prestation de soins est en émergence: le suivi systématique des clientèles, parfois appelé gestion de cas (MEQ, 1996, p.49).

Bref, la transformation des besoins et des attentes de la population, l'évolution des technologies et les changements d'orientation dans la distribution des soins et des services semblent avoir des incidences importantes sur le travail du personnel en soins infirmiers, que ce travail soit effectué en établissement ou à domicile. Ils transforment les méthodes de travail, diversifient les procédés utilisés et multiplient les activités (MEQ, 1996 p.51).

[...] on ne peut sous-estimer l'importance du virage ambulatoire. Celui-ci contribue à modifier le rôle exercé par les équipes de soins infirmiers dans la mesure où il est possible que les personnes traitées en établissement présentent des problèmes de 
santé plus aigus et nécessitent des soins plus complexes. Par ailleurs, l'accroissement des services à domicile risque d'entraîner de nouvelles pratiques pour le personnel infirmier et des modifications importantes dans la façon d'aborder leur travail dans la mesure où de nouvelles tâches peuvent s'ajouter (MEQ, 1996, p.4).

Parmi les nouveaux besoins exprimés par les organismes, celui d'apprendre revient plus souvent dans leur discours. En effet, les organismes accordent une grande importance à l'enseignement et à la communication en lien avec la pratique infirmière de l'avenir axée davantage sur le travail communautaire où l'usager est encouragé à prendre sa santé en charge:

[...] le nursing orienté vers le mode de vie en santé doit fournir un environnement dans lequel les individus, les familles ou les groupes peuvent apprendre. Cela signifie que le nursing façonne la structure de l'apprentissage: il permet l'accès à la connaissance et à l'information; il favorise des situations dans lesquelles on peut discuter, partager et travailler avec d'autres qui poursuivent des buts semblables (OIIQ, 1989, p.9).

[...] la dimension enseignement est devenue omniprésente dans le travail de l'infirmière. On leur demande donc d'avoir certaines habiletés de communication, des capacités d'animation de groupes et certaines connaissances sur les processus d'apprentissage (MEQ, 1996, p.58).

Si l'ensemble des organismes est d'accord pour dire qu'il y a une transformation du rôle de l'infirmière, l'OIIQ, pour sa part, est beaucoup plus précise dans la description des multiples rôles que l'infirmière est appelée à jouer:

Les infirmières fonctionnent dans une variété de rôles tels: soignante, dispensatrice de soins de première ligne à un individu, éducatrice pour la santé des groupes, conseillère en matière de santé, administratrice de programme de soins. Parmi les divers rôles reliés à la poursuite des buts de promotion et de protection de la santé axés sur l'autodétermination de la clientèle, il y a ceux d'agent de changement, de personne-ressource, d'avocate, de partenaire, de coordonnatrice, d'agent de continuité, de pivot, d'animatrice d'équipe de travail ou de divers types de groupes, 
d'enseignante, de soutien psychosocial, d'agent de référence, d'administratrice, de chercheure et de membre d'une profession (OIIQ, 1989, p.11).

L'analyse nous révèle également que les habiletés en communication de l'infirmière, dans le cadre de la réforme de la santé, lui permettront d'occuper une place privilégiée au sein de l'équipe interdisciplinaire et/ou multidisciplinaire. Par ailleurs, la Fédération des infirmières et des infirmiers du Québec et le MEQ reconnaissent que d'autres tâches liées à la coordination et à la planification devront également être assumées par les infirmières, afin d'assurer la collaboration entre les différents acteurs impliqués du système:

L'infirmière sera également l'intervenante privilégiée pour favoriser la continuité et la complémentarité des soins et services par une communication efficace entre les différents intervenants, une collaboration étroite entre les membres de l'équipe interdisciplinaire ainsi que la coordination de leurs interventions (ADRSI, 1993, p.48).

Les nouvelles tâches des infirmières et infirmiers sont liées à la planification et à la coordination de l'épisode de soins et sont accomplies en étroite collaboration avec une équipe multidisciplinaire (MEQ, 1996, p.58).

Ces discours nous ramènent à la dimension «autonomie» de l'infirmière qui est au centre de la problématique de la professionnalisation. À ce sujet, nous aimerions rappeler que pour l'OIIQ (1989), l'autonomie signifie la liberté et la responsabilité dans la prise de décisions. Ainsi, l'autonomie représente la condition essentielle d'une pratique «professionnelle». Les concepts d'autonomie et de responsabilités ont été clairement identifiés dans chacun des discours analysés. En effet, tous les organismes reconnaissent que les nouvelles pratiques requièrent davantage d'autonomie et exigent un sens des responsabilités accru. Pour le MEQ, l'autonomie professionnelle, en plus de faire référence au jugement clinique de l'infirmière, suppose également un encadrement différent de la pratique en milieu hospitalier: 
[...] l'infirmière ou l'infirmier doit dorénavant être capable de «penser» et de mettre en œuvre les soins infirmiers plutôt que d'être centré sur l'exécution d'une ordonnance. Du fait que l'encadrement offert au personnel infirmier par le milieu hospitalier est de plus en plus léger, les exigences le plus souvent mises en relief sont celles qui sont liées à la manifestation d'initiative, au sens des responsabilités et à l'autonomie professionnelle (MEQ, 1996, p.58).

L'autonomie des infirmières et des infirmiers prend une place de plus en plus importante dans leur pratique professionnelle (MEQ, 1996, p.48).

Pour la FIIQ, il s'agit plutôt d'une reconnaissance de l'autonomie dans la pratique infirmière: «C'est la dimension la plus autonome de la profession qui pourrait enfin être mise pleinement à contribution» (FIIQ, 1995, p.31).

Les discours rapportent que le contexte social actuel, plus exigeant, nécessitera une plus grande autonomie de l'infirmière. Cette autonomie «étendue» pourrait s'obtenir en augmentant les connaissances et les compétences des infirmières au cours de leur formation. Par l'analyse du contexte de formation, nous tenterons de faire ressortir la position des différents organismes, quant au niveau de formation initiale de l'infirmière, dans un contexte de mutation des soins de santé.

\subsection{Contexte de formation}

Comme le démontre l'analyse des contextes social et professionnel, la pratique infirmière subira des changements majeurs avec la réorganisation du réseau de la santé. Selon l'OIIQ et le MEQ, la formation des infirmières doit répondre aux besoins futurs en matière de la santé:

Il faut donc prévoir répondre aux besoins de santé et de soins infirmiers qui surviendront ultérieurement. Cela signifie que la formation doit être prospective; elle 
doit s'assurer que la formation initiale donnée aux infirmières puisse répondre aux besoins qui se dessinent dès aujourd'hui et dont nous commençons à peine à prévoir l'ampleur et la complexité (OIIQ, 1989, p.5).

[...] la philosophie et les objectifs des programmes de formation infirmière doivent désormais refléter l'importance accordée à la promotion de la santé et au bien-être des individus, des familles, des groupes et de la société (OIIQ, 1989, p.12).

Dans un contexte où l'on assiste à une restructuration des principales fonctions dans le domaine des soins infirmiers, il importe de s'assurer de l'adéquation des programmes de formation par rapport aux besoins de la main-d'œuvre et du marché du travail (MEQ, 1996, p.140).

Rappelons qu'au Québec, malgré le dépôt du rapport Rodger en 1981 qui recommandait une formation de base de niveau collégial, il existe toujours deux programmes de formation en soins infirmiers de niveau supérieur, l'un technique ${ }^{5}$ de niveau collégial et l'autre professionnel de niveau universitaire.

L'accès à la formation collégiale en soins infirmiers est possible après l'obtention d'un diplôme d'études secondaires et se poursuit pendant six sessions. Le nombre d'unités ${ }^{6}$ total est de $91,2 / 3$ correspondant à 2775 heures d'enseignement, incluant la formation fondamentale et spécifique.

Le programme de formation en soins infirmiers du cégep est considéré comme la première étape d'un programme de formation continue. Il prépare les étudiantes à l'exercice de la profession en milieu hospitalier. L'infirmière diplômée est en mesure de prodiguer les soins infirmiers que requièrent des individus dans des situations

5 Le nom du programme a été modifié: Soins infirmiers a remplacé Techniques infirmières et ce, en vue de mieux refléter les orientations du programme d'études. Le titre de technicienne semble comporter une connotation plutôt imprécise et restrictive: elle n'intègre pas le service d'aide et de sollicitude qui caractérise l'interaction entre l'infirmière et son client, lequel ne relève pas de la technique (Goulet, O.; 1993, p.172).

6 L'unité correspond à 45 heures d'activités de formation pouvant comprendre des leçons magistrales, des travaux pratiques d'atelier ou de laboratoire. (LEGENDRE, R. (1993). Dictionnaire actuel de l'éducation, $2^{e}$ édition, Montréal: Guérin, p. 1392). 
courantes relevant de la promotion et du recouvrement de la santé (Potter, 1990, p.14).

Dans le cas de la formation initiale universitaire, pour y avoir accès, l'étudiante doit avoir complété une formation de quatre sessions au programme de sciences de la nature à l'enseignement collégial; formation totalisant 58,67 unités. La formation universitaire répartie sur six sessions compte entre 90 et 111 crédits $^{7}$ représentant au-delà de 4000 heures d'enseignement.

Le programme d'études universitaires de $1^{\text {er }}$ cycle prépare les étudiantes à exercer la profession d'infirmière dans différents milieux et à différents paliers de soins, et à œuvrer pour la promotion et le recouvrement de la santé. Le programme d'études universitaires vise à rendre l'infirmière capable d'appliquer la méthode de résolution de problèmes à des situations de santé simples ou complexes et dans une perspective de soins infirmiers à court et à long terme» (Potter, 1990,p.14).

Rappelons que ces deux formations de niveau supérieur donnent accès au même droit de pratique.

Le MEQ (1996) rapporte les commentaires suivants concernant la même fonction que jouent les diplômées de ces deux niveaux de formation:

Car les bachelières et les techniciennes ont des titres d'emploi distincts mais, à ce jour, ces titres correspondent à la même fonction de travail, celle d'infirmière et d'infirmier. Certaines personnes ont d'ailleurs déploré le fait que, sur le terrain, les tâches de la bachelière et du bachelier ne se distinguent pas de façon marquée de celles exercées par la technicienne et le technicien (MEQ, 1996, p.133).

7 Le crédit est une unité de mesure qui sert à évaluer la charge de travail dans un cours. Il correspond normalement à 45 heures de travail. Cela comprend à la fois la présence en classe et le travail personnel. (UNIVERSITÉ LAVAL (1997). Sciences de la santé. Les programmes de premier cycle, Québec: Bureau du registraire, p. 4). 
Nous croyons, effectivement, que l'absence de description au niveau des tâches spécifiques aux titres d'emploi d'infirmière diplômée et d'infirmière bachelière contribue à semer la confusion autant dans les milieux de travail et de formation, qu'auprès des usagers.

D'ailleurs, il y a déjà deux décennies que cette situation ambiguë persiste. Certaines recommandations concernant la formation infirmière avaient été faites par le comité Rodger comme le rapporte l'OIIQ:

Suite à une demande expresse du ministère de l'Éducation en 1978, le Comité Rodger a eu pour mandat d'effectuer une étude approfondie sur la situation d'ensemble des programmes de formation infirmière. Le rapport fut publié en juin 1981. Il identifiait plusieurs problèmes vécus dans la pratique infirmière et présentait des recommandations dont, entre autres, le choix de la formation technique au cégep comme seule voie d'entrée à la profession, l'allongement d'un an du cours de techniques infirmières et l'abolition de la formation professionnelle initiale universitaire (OIIQ, 1989, p.19).

Cette double formation, comme le rapporte la FIIQ, est spécifique à la discipline des soins infirmiers. Elle précise que pour les autres professionnels de la santé (physiothérapeutes, ergothérapeutes, diététistes, etc.) la seule formation donnant accès au droit de pratique est de niveau universitaire: «De toutes les professionnelles du domaine de la santé, l'infirmière est la seule qui puisse choisir entre deux niveaux de formation initiale» (FIIQ, 1995, p.12).

La FIIQ, en désaccord avec cette double formation, considère que la formation collégiale peut contribuer à former des infirmières aptes à dispenser des soins de santé: «D'ailleurs, la formation qu'ont reçue jusqu'à maintenant les infirmières, semble avoir gé- 
néré un personnel efficace qui dispense des services de santé dont la population se dit satisfaite» (FIIQ, 1995, p.15).

La position des autres organismes consultés est assez partagée en ce qui concerne le niveau de formation initiale de l'infirmière. En effet, l'OIIQ et l'ADRSI sont en faveur d'une formation initiale universitaire. Selon ces organismes, une formation universitaire contribuerait à l'avancement de la profession:

La prise de position de l'Ordre en faveur de la formation initiale universitaire pour l'admission à la profession en l'an 2000 constitue un changement majeur dans l'évolution de la profession infirmière au Québec [...] (OIIQ, 1989, p.45).

L'infirmière de l'avenir devra être formée à l'université. L'accès à la profession devra passer par l'acquisition d'un baccalauréat de base en sciences infirmières (ADRSI, 1993, p.25).

Or, le déplacement important des ressources vers la première ligne et l'accroissement de la complexité des cas en milieu hospitalier exigeront des infirmières une formation initiale universitaire afin qu'elles soient aptes à remplir le nouveau mandat qui leur est confié [...] (OIIQ, 1996, p.53).

Pour défendre sa position, l'Ordre des infirmières s'appuie sur le mouvement de professionnalisation qui caractérise ce métier. Si le problème actuel est la valorisation de la profession et le mouvement de professionnalisation, la formation universitaire semble en effet une voie prometteuse.

Il en va autrement pour la FIIQ pour qui la formation initiale universitaire ne permettrait en aucun cas de régler les problèmes actuels liés aux contextes social, économique et professionnel dans lesquels la profession s'exerce: 
Quant à la formation initiale strictement universitaire pour accéder à la profession, elle ne réglerait pas les problèmes avec lesquels la profession est aux prises et elle réduirait l'accessibilité à la profession de plusieurs manières (FIIQ, 1995, p.43).

Et d'un point de vue économique, l'accessibilité serait réduite du fait que la formation universitaire est plus dispendieuse pour les étudiantes, puisqu'elle prolonge la période scolaire de deux ans tout en amputant les revenus (FIIQ, 1995, p.43).

[...] le cheminement qui conduit au baccalauréat en formation initiale s'avère plus onéreux que la formation initiale au collégial (FIIQ, 1995, p.18).

L'accessibilité aux études universitaires ne nous semble pas aussi problématique que le rapporte la FIIQ. Concernant les obstacles économiques à l'accès aux études, nous croyons que les étudiantes inscrites dans des programmes universitaires pourraient avoir recours au programme de prêts et bourses du gouvernement, comme le fait la majorité des étudiants inscrits dans des programmes d'études supérieures. Le Programme de prêts et bourses du ministère de l'Éducation est offert aux personnes qui désirent poursuivre des études post-secondaires et dont les ressources financières sont insuffisantes (Service régional d'admission du Montréal métropolitain, 1999, Guide pratique des études collégiales, p.363).

De plus, pour la FIIQ, l'accès géographique représente un autre obstacle aux études universitaires:

Effectivement, bon nombre de candidates ne peuvent y parvenir faute de moyens financiers et dû à un accès géographique limité (FIIQ, 1995, p.18).

D'un point de vue géographique, l'accessibilité serait limitée à certaines régions contrairement à la formation collégiale qui est dispensée dans quarante-deux cégeps (FIIQ, 1995, p.43).

Du point de vue géographique, nous croyons que les études universitaires, depuis plusieurs années, sont accessibles dans toutes les régions du Québec par le réseau de l'U- 
niversité du Québec constitué de six universités à vocation générale: l'Université du Québec à Montréal (UQAM), l'Université du Québec à Chicoutimi (UQAC), l'Université du Québec à Hull (UQAH), l'Université du Québec à Rimouski (UQAR) et l'Université du Québec à Trois-Rivières (UQTR) et l'Université du Québec en Abitibi-Témiscamingue (UQAT). Il nous apparaît peu probable, à la lumière de ce constat, qu'une formation de base universitaire réduise l'accès à la profession infirmière pour des questions géographiques.

Les arguments sur lesquels les organismes s'appuient pour choisir le niveau de formation de base en soins infirmiers sont très variés. Le MEQ n'affiche pas sa position quant au niveau de formation de base en soins infirmiers. En effet, ce sont les ordres professionnels qui ont le mandat de réglementer la formation et l'entrée à la profession. Cependant, le MEQ dénonce la confusion et le chevauchement dans les programmes actuels d'enseignement en soins infirmiers et indique que les deux niveaux d'enseignement ont comme principal objectif la formation d'infirmières généralistes donnant accès au droit de pratique:

Il reste que l'objectif premier des deux programmes est d'assurer la formation d'infirmière et d'infirmier. Mises à part, les dimensions précédentes qui sont spécifiques à l'enseignement universitaire, des similitudes importantes caractérisent les deux programmes (MEQ, 1996, p.132).

Les deux programmes (collégial et universitaire) prétendent former la clientèle étudiante à la même fonction de travail, même si les programmes d'enseignement sont différents, puis chaque ordre ignore le programme offert par l'autre (MEQ, 1996, p.139).

Plusieurs aspects sont communs aux deux programmes (MEQ, 1996, p.131).

Dans la mesure ou les deux ordres d'enseignement offrent la formation de base et se veulent la porte d'accès à la profession, il apparaît clairement que ces programmes ne sont pas offerts en continuité mais plutôt en duplication (MEQ, 1996, p.139). 
Les nuances entre les objectifs des deux programmes nous semblent difficiles à cerner. Toutefois, quelques aspects de la pratique nous apparaissent plus clairs à la lecture des textes que nous avons parcourus. Ainsi, le Document d'accompagnement du programme soins infirmiers 180-01 précise que l'infirmière diplômée au collégial doit poursuivre sa formation pour accomplir d'autres rôles liés à l'approche collective, l'encadrement du personnel, la spécialisation, l'administration et la recherche (DGEC, p.8, 1987), alors que quelques années d'expérience et une formation additionnelle donneront accès à des domaines particuliers des soins infirmiers ou à une carrière en administration, en consultation, en enseignement ou en recherche pour l'infirmière bachelière (Université Laval, p.19, 1997).

À partir du fait que les deux niveaux d'études supérieures forment des infirmières généralistes, il est plus aisé de comprendre la position de la FIIQ, concernant le niveau de formation initiale donnant accès au droit de pratique. Pour la Fédération, le programme collégial en soins infirmiers semble mieux répondre aux défis d'un environnement en transformation, en plus d'être financièrement plus accessible que le programme universitaire. La FIIQ croit que le programme collégial en soins infirmiers est en mesure de répondre aux demandes actuelles:

Le programme collégial de formation en soins infirmiers, actualisé en 1988, répond mieux aux défis d'un environnement en transformation ainsi qu'aux exigences du marché du travail, en demeurant socialement plus économique et plus accessible aux étudiantes (FIIQ, 1995, p.42).

La FIIQ ne précise pas à quels défis elle fait référence. Or, les défis sont multiples actuellement autant dans le domaine de la santé que dans celui de l'éducation. Comment la FIIQ peut-elle tenir un tel discours dans le contexte social actuel où le principal défi des infirmières est l'adaptation continue à des milieux de travail en mutation? La FIIQ serait- 
elle en désaccord avec la révision du programme collégial en soins infirmiers? Le programme actuel a été élaboré au début des années 80 , c'est-à-dire bien avant les réformes de la santé et de l'éducation. Il nous semble utopique de croire que le programme élaboré en 1987 réponde aux nouvelles demandes sociales en soins de santé. De toute façon, il nécessite sûrement quelques ajustements quoiqu'en pense la FIIQ.

À l'encontre de la FIIQ, l'ADRSI prétend que la formation universitaire a déjà fait ses preuves et mérite d'être encouragée. Alors que pour la FIIQ, la formation collégiale est suffisante pour répondre aux exigences du marché du travail, l'ADRSI se positionne en faveur d'une formation universitaire pour répondre aux mêmes exigences:

[...] la contribution de la formation universitaire a été reconnue et l'accès à cette formation a été encouragée (ADRSI, 1993, p.4).

L'accès à la profession infirmière devra passer par l'acquisition d'un baccalauréat de base en sciences infirmières. Nous croyons que cette disposition est nécessaire pour répondre aux besoins des usagers, pour le progrès des soins infirmiers et leur adaptation aux tendances qui se manifestent (ADRSI, 1993, p.25).

Lorsque l'ADRSI parle de progrès en soins infirmiers, nous pensons qu'elle fait référence à la recherche infirmière. La recherche, pierre angulaire des programmes de formation universitaire, favorise le développement du domaine et permet d'accroître l'autonomie professionnelle.

La position de l'OIIQ est très claire en ce qui concerne la formation initiale de l'infirmière: elle doit être universitaire. Déjà en 1982, l'Ordre prenait position pour la formation initiale universitaire dans le but d'acquérir une plus grande autonomie professionnelle. L'analyse du discours de l'Ordre fait ressortir clairement la notion d'autonomie professionnelle dans le nouveau paradigme de santé et bien-être: 
[...] la plupart des responsables de programmes en soins infirmiers universitaires ont révisé les programmes de baccalauréat de manière à former une professionnelle généraliste qui se préoccupe de la santé des individus, des familles et des collectivités, qui cherche à répondre aux besoins de la population, et qui agisse de façon autonome (OIIQ, 1989, p.21).

[...] les exigences de la formation (universitaire) proviennent principalement du fait que c'est d'un savoir d'une nature différente dont la profession d'infirmière a besoin et que la profession d'infirmière développe. Ce savoir est de niveau professionnel, et de calibre universitaire. Il porte sur la conception, la planification et l'organisation des soins, tout autant que sur leur dispensation. Il reflète la maîtrise d'une réalité professionnelle autonome (OIIQ, 1989, p.31).

Les fonctions dévolues à l'infirmière formée à l'université, de même que les différents aspects de son rôle, rejoignent la pensée de l'OMS lorsqu'elle situe les infirmières par rapport aux soins de santé primaires (OIIQ, 1989, p.23).

À notre avis, l'argumentation de l'OIIQ s'appuie sur le mouvement de professionnalisation qui caractérise le métier d'infirmière.

Pour le MEQ, l'importance réside non pas dans le niveau de la formation de base en soins infirmiers, mais dans l'adéquation entre la formation et les demandes sociales:

Dans un contexte où l'on assiste à une restructuration des principales fonctions dans le domaine des soins infirmiers, il importe de s'assurer de l'adéquation des programmes de formation par rapport aux besoins de la main-d'œuvre et du marché du travail (MEQ, 1996, p.140).

Toutefois, le MEQ reconnait que certaines tâches exigent une formation de niveau universitaire:

L'approche collective en regard de la promotion de la santé et de la prévention de la maladie, l'encadrement du personnel infirmier, la spécialisation en soins infirmiers ainsi que les domaines de l'administration, de l'éducation et de la recherche nécessitent une formation universitaire (MEQ, 1996, p.132). 
Parmi les organismes consultés, seul l'OIIQ énonce les qualifications que l'infirmière devrait acquérir en cours de formation pour répondre aux demandes actuelles et futures dans le domaine de la santé. Au centre de ces qualifications, la notion d'autonomie prend tout son sens puisqu'elle permet à l'infirmière de développer d'autres habiletés connexes telles que la prise de décision, la résolution de problèmes et l'habileté à porter un jugement:

Nous voulons donc que la formation de l'infirmière soit suffisamment poussée pour lui permettre d'être autonome dans ces interventions, pour lui permettre d'agir en collaboration avec les autres professionnels de la santé et pour lui permettre de répondre à la complexité des besoins de la clientèle (OIIQ, 1989, p.5).

[...] la formation infirmière appropriée requiert l'utilisation d'une vaste étendue de connaissances, la maîtrise d'habiletés reliées au rapport d'égalité, au support du processus de prise de décision reposant sur des systèmes de valeurs différents de celui de l'infirmière, et un degré élevé de conscience sociale et d'engagement professionnel (OIIQ, 1989, p.12).

Les infirmières devront être formées pour la pratique future qui sera plus complexe et spécialisée qu'actuellement. L'orientation du système de soins vers la santé, l'accent sur la promotion de la santé et le déplacement du système de santé vers la communauté, créent des impératifs sur les programmes de formation et un réalignement fondamental de la formation initiale car les infirmières doivent être préparées pour exercer dans un système de santé basé sur la communauté (OIIQ, 1996, p.48).

La résolution de problèmes, la prise de décision et l'habileté de porter un jugement clinique sont des habiletés importantes et essentielles à développer [...] (OIIQ, 1996, p.48).

Concernant les nouvelles connaissances, nous pensons que l'OIIQ compte sur la rigueur intellectuelle inculquée par des études universitaires pour encourager les infirmières à demeurer au fait des nouvelles connaissances permettant d'améliorer leur pratique: 
[...] pour pouvoir accompagner le développement de la technologie, cela exigera l'acquisition de nouvelles connaissances et habiletés de la part de l'infirmière [...] (OIIQ, 1996, p.50).

[...] devront posséder une bonne connaissance des lois et règlements et ressources afin d'assurer l'assistance nécessaire [...] (OIIQ, 1996, p.50).

[...] les infirmières ont besoin de comprendre le processus du vieillissement et les problèmes de santé de la personne âgée (OIIQ, 1996, p.50).

Ce sont les habiletés en communication qui permettront surtout à l'infirmière d'élargir son champ d'action. Il nous semble évident que pour l'OIIQ, l'infirmière doit être présente à tous les paliers du domaine de la santé, c'est-à-dire auprès de l'usager, au cœur de l'équipe interdisciplinaire, en recherche et sur le plan politique:

Les infirmières devront posséder des habiletés leur permettant de favoriser la collaboration interdisciplinaire [...] (OIIQ, 1996, p.49).

Les infirmières devront posséder les habiletés pour assister les usagers à avoir de bonnes habitudes de vie et à assumer un rôle actif dans le maintien de leur santé (OIIQ, 1996, p.50).

[...] être capables de s'impliquer et d'intervenir sur le plan politique afin de provoquer des changements, d'influer sur le système de santé et de supporter la mise en place de politiques assurant la santé et le mieux-être (OIIQ, 1996, p.50).

[...] devront faire preuve d'ouverture face à la recherche (OIIQ, 1996, p.48-51).

Il sera important de développer un sens des responsabilités [...] (OIIQ, 1996, p.50).

Comme le soulignent les organismes, la formation infirmière doit répondre aux besoins et demandes en soins de santé. Certaines études citées rapportent la satisfaction de la communauté et des employeurs quant au travail des infirmières. Il est à noter que ces études sont rapportées par les tenants de la formation collégiale en soins infirmiers: 
D'ailleurs la formation qu'ont reçue jusqu'à maintenant les infirmières, semble avoir généré un personnel efficace qui dispense des services de santé dont la population se dit satisfaite (FIIQ, 1995, p.25).

Le taux de satisfaction des employeurs à l'égard du personnel infirmier issu du programme Soins infirmiers est relativement élevé (MEQ, 1996, p.116).

En effet, en 1994, 92,4\% des 131 employeurs interrogés se sont dits satisfaits du rendement global des titulaires de diplôme en Soins infirmiers après six mois d'exercice contre $6,9 \%$ qui en sont insatisfaits (MEQ, 1996, p.116).

L'enquête Relance, effectuée en 1994, qui rapporte la satisfaction des employeurs quant au rendement des finissantes en soins infirmiers de niveau collégial, vient confirmer au MEQ l'adéquation du programme Soins infirmiers distribué dans les 42 collèges de la province. Le rapport de cette enquête est très positif si on le compare à celui de 1984 publié par la firme SOBECO qui dénonçait de l'insatisfaction face aux programmes d'enseignement collégial et universitaire:

[...] que la majorité des établissements qualifiait d'inadéquate la formation scolaire actuelle des infirmières diplômées des niveaux collégial et universitaire (OIIQ, 1989, p.32).

Le rapport ne relève pas les aspects précis des faiblesses théoriques ou pratiques que l'on reproche aux jeunes diplômées (OIIQ, 1989, p.32).

Pourtant, si elle semble satisfaire les employeurs et la communauté, la formation des infirmières ne semble pas répondre entièrement aux attentes de l'Ordre professionnel qui est en faveur d'une formation initiale universitaire:

Malgré un engagement significatif de la profession d'infirmière à la prévention de la maladie et à la promotion de la santé, l'emphase dans la majorité des programmes de formation en soins infirmiers a porté (et porte encore) principalement sur les connaissances et les habiletés nécessaires pour soigner les individus lors de maladies ou d'états de crise. Encore de nos jours, l'enseignement et la pratique des soins infirmiers se font en étroite symbiose avec l'hôpital et dans un contexte où les soins curatifs dominent (OIIQ, 1989, p.33). 
Effectivement, le programme d'enseignement collégial en soins infirmiers prévoit l'étude de plusieurs problèmes prioritaires de santé chez l'adulte et l'enfant. De plus, la majorité des laboratoires-cliniques ont lieu en centre hospitalier, c'est-à-dire auprès d'individus devant recevoir des soins curatifs. Or, la nouvelle définition du concept de santé et de bien-être amène une transformation du rôle de l'infirmière qui devra, en plus de dispenser des soins, être en mesure d'aider les individus à prendre leur santé en charge.

Malgré les divergences d'opinion des organismes consultés lors de États généraux de l'éducation tenus en 1995 et des États généraux de la profession infirmière tenus en 1996, le MEQ annonçait en 1996, suite à des analyses de situation de travail, un nouveau programme collégial en soins infirmiers élaboré selon l'approche par compétences.

L'approche par compétences, selon Goulet, J.P. (1994), constitue la pièce de résistance pédagogique de la réforme de l'éducation. Le ministère a décidé d'utiliser cette méthode pour réviser les programmes de formation à partir desquels seront issus les devis ministériels (Goulet, J.P., 1994). Cet auteur rapporte que les visées éducatives de cette approche sont centrées sur des apprentissages pertinents et significatifs qui sont en lien avec des situations complexes et que les élèves rencontreront au-delà de leur programme d'études. Selon Goulet, J.P. (1994), ces apprentissages paraitront significatifs puisqu'ils seront ancrés dans des situations de vie personnelle, sociale et professionnelle plutôt que seulement dans le contexte scolaire.

En soins infirmiers, c'est en octobre 1999 que la Direction générale de la formation professionnelle et technique présentait la première version du Projet de programme d'études en soins infirmiers. Dans le cadre de cette recherche, nous avons utilisé la version finale, soit celle adressée aux directeurs et directrices des études des cégeps de la province en décembre 2000. 
Avant de présenter notre travail d'analyse du document Projet de programme d'études en soins infirmiers élaboré selon l'approche par compétences, nous présentons une synthèse de l'analyse de contenu effectuée à partir des textes officiels.

\subsection{Synthèse des résultats de l'analyse de contenu}

Il ressort des textes qui ont servi à l'analyse une certaine similitude dans les discours quant aux descriptions des contextes social et professionnel. En effet, les organismes s'entendent pour dire que les facteurs à la base des transformations du réseau de la santé découlent en grande partie de la crise des finances publiques. L'accroissement des coûts liés aux soins de santé, force l'État à adopter de nouveaux modes de distribution de soins qui permettront de mieux répartir les ressources allouées aux soins de santé et aux services sociaux, en favorisant la croissance des organismes communautaires. La nouvelle philosophie de soins adoptée par le gouvernement, davantage axée sur la promotion de la santé et la prévention de la maladie, place le citoyen au cœur du processus décisionnel et le responsabilise davantage face à santé. La croissance des organismes communautaires permettra de répondre aux nouveaux besoins d'une population qui désire être mieux informée et davantage consultée sur les décisions qui concernent les soins de santé. Cette nouvelle philosophie amène également des changements considérables au niveau de la distribution des soins. Le rôle professionnel de l'infirmière est appelé à changer dans un avenir assez rapproché. Dans la communauté, les nouveaux modes de distribution de soins requièrent de la part des infirmières des connaissances élargies, une plus grande autonomie, un sens élevé de responsabilité, des aptitudes en gestion, en coordination, en enseignement et en animation de groupe. 
C'est au niveau de la formation que les discours des organismes diffèrent. Il n'y a pas de consensus quant au niveau de formation initiale en soins infirmiers à privilégier. L'OIIQ et l'ADRSI prétendent qu'il serait souhaitable qu'il n'y ait qu'un seul niveau de formation initiale. Selon eux, seule une formation de niveau universitaire peut permettre l'acquisition des compétences requises pour répondre à l'ensemble des demandes actuelles en soins de santé. La FIIQ et le MEQ allèguent que la formation collégiale en soins infirmiers demeure la formation initiale de choix pour les infirmières. Ils déclarent que les employeurs sont très satisfaits du rendement des finissantes en soins infirmiers des collèges de la province. Si la FIIQ affiche clairement son désaccord avec une formation initiale de niveau universitaire, le MEQ se garde bien, dans son discours, de prendre franchement position quant aux niveaux de formation initiale en soins infirmiers. Il reconnaît cependant que certaines compétences ne peuvent être acquises que par la poursuite d'études universitaires. Non seulement le MEQ ne tranche pas la question, mais il maintient la formation initiale de niveau collégial ainsi que celle de niveau universitaire alors qu'il dénonce, lui-même, les chevauchements. Dans ce sens, il propose un nouveau programme, selon l'approche par compétences, malgré que l'ancien semblait répondre aux exigences des employeurs et prescrit un continuum de formation en soins infirmiers sur cinq ans dans le but de faciliter l'accessibilité aux études universitaires: trois ans au collège avec l'accès au droit de pratique et deux ans à l'université pour acquérir un baccalauréat en sciences infirmières. 


\section{CHAPITRE V}

\section{Analyse du programme de formation collégiale en soins infirmiers élaboré selon l'approche par compétences}

Il nous semble important de préciser que l'orientation axée sur les compétences, comme le rapporte Touzin (1994), fait suite à l'adoption en décembre 1990, de la Direction générale de l'enseignement collégial de définir les programmes de formation professionnelle selon cette approche.

Étant responsable de la formation collégiale en soins infirmiers depuis 1967, le MEQ, suite aux analyses de situations de travail effectuées en 1996, propose un nouveau programme de soins infirmiers de niveau collégial: le programme Soins infirmiers 180.A0, élaboré selon l'approche par compétences. Dans la foulée des recommandations des États généraux sur l'éducation qui ont débuté au printemps 1995, la révision des programmes d'études du ministère de l'Éducation du Québec se fait selon l'approche par compétences. Cette approche est axée sur l'apprentissage plutôt que sur l'enseignement contrairement aux approches traditionnelles. L'approche par compétences aurait pris naissance aux États-Unis vers la fin des années 60 et le début des années 70 (Tremblay, 1990: voir Touzin, 1994). La compétence, rapporte Laferrière (1994), met l'accent sur la capacité de l'individu à faire quelque chose plutôt que sur son aptitude à démontrer ses connaissances, l'intervention se situant davantage au niveau du processus qu'au niveau de la 
transmission de connaissances. Les nouveaux programmes ministériels étant élaborés selon l'approche par compétences, il nous apparaît essentiel, dans un premier temps, de définir la notion de «compétence» qui semble polysémique et imprécise.

Dans le domaine de l'éducation, plusieurs chercheurs ont élaboré une définition de la notion de compétence. Il semble, selon Tremblay (1990: cité dans Lavoie et Painchaud, 1993), qu'il existe tout près d'une centaine de définitions du mot «compétence» dans les écrits spécialisés sur le sujet. Selon BIT (1987: cité dans Legendre, 1993, p.223), la compétence est «l'aptitude à exercer efficacement un métier, une fonction ou certaines tâches spécifiques, avec toutes les qualifications requises à cet effet». Laferrière (1994) désigne la compétence comme «étant une habileté psycho-sensori-motrice ou un comportement socio-affectif et non comme une habileté cognitive seulement» (p.4). Nous croyons, en effet, qu'aux connaissances doivent s'ajouter des habiletés et des attitudes. Pour Perrenoud (1995), «les compétences renvoient à des savoir-faire de haut niveau qui exigent l'intégration de multiples ressources cognitives dans le traitement de situations complexes» (p.6). Selon Tremblay :

[...] la compétence est un concept général qui englobe la faculté d'utiliser ses capacités et ses connaissances dans des situations nouvelles à l'intérieur de son champ professionnel. Elle intègre également l'organisation et la planification du travail, l'innovation et la capacité de s'adapter à des activités non routinières. Finalement, elle inclut les qualités personnelles nécessaires pour interagir efficacement avec les collègues, la direction et les clients (1990: voir Lavoie et Painchaud, 1993, p.17).

Cette définition de la compétence, à la fois descriptive et englobante, nous semble être en lien avec l'expérience. Nous croyons que l'organisation, la planification du travail et la capacité de s'adapter à des activités non routinières sont des aspects de la compétence qui s'acquièrent avec le temps. 
Laferrière (1994) distingue les compétences générales, «qui dépassent l'utilité, la rentabilité et la performance pour viser une formation globale et intégrée», des compétences professionnelles «qui doivent être transférables à une variété de contextes» (p.5). Il faut éviter, souligne Laferrière (1994,p.4-5), «une conception réductionniste de la compétence en la réduisant à une tâche précise commandée par une exigence spécifique du travail en milieu industriel». D'ailleurs, Barbès (1990) définit la compétence sous la perspective professionnelle:

[...] la compétence professionnelle est la qualité (du professionnel) qui exerce sa profession selon les normes d'exercice, c'est-à-dire qui, possédant les connaissances scientifiques et professionnelles, le jugement et les habiletés nécessaires, les attitudes et les valeurs appropriées, s'acquitte adéquatement des tâches inhérentes à ses fonctions (de professionnel) dans un rôle donné (voir Lavoie et Painchaud, 1993, p.17).

Cette définition se rapproche de celle de l'OIIQ qui définit la compétence comme étant:

[...] la qualité d'une infirmière ou d'un infirmier qui exerce sa profession selon les normes d'exercice, c'est-à-dire qui, possédant les connaissances scientifiques et professionnelles, le jugement et les habiletés nécessaires, s'acquitte adéquatement des tâches inhérentes à ses fonctions d'infirmière ou d'infirmier dans un rôle donné (1980, p.5).

L'OIIQ (1980) précise qu'il ne s'agit pas seulement de connaissances et d'habiletés mais aussi d'attitudes et de jugement nécessaires à l'exercice de la profession. D'ailleurs, pour Gauthier et Tardif (2000),

[...] l'acquisition de compétences professionnelles se réfère à un processus multidimensionnel où interviennent des aspects identitaires (personnalité du travailleur, conception de soi, sentiment personnel de compétence, etc.), sociaux (évaluation par les pairs, les autorités, les clients, etc.), temporels (compétences découlant de l'histoire de vie, de la formation professionnelle et de l'évolution de la carrière) et 
pratiques (développement de routines et de procédures concrètes d'action liées à la réalisation de tâches précises) (p.15).

Ils précisent: «Travailler avec des êtres humains nécessite l'acquisition d'un large spectre de compétences qui ne se réduisent jamais à des savoir- faire techniques) (p.15).

En soins infirmiers, le modèle d'acquisition de compétences qui s'applique est celui de Dreyfus (1980, cité dans Benner, 1995). Ce modèle suppose que l'infirmière évolue selon cinq stades successifs dans le but d'acquérir et de développer une compétence: novice, débutant, compétent, performant et expert. Une définition de chacun de ces niveaux de compétence, du modèle de Dreyfus, nous permettra de vérifier le sens attribué, dans cette échelle, à la notion de «compétence». Ainsi, selon Dreyfus:

L'infirmière novice n'a aucune expérience des situations auxquelles elle risque de se trouver confrontée et doit recevoir des règles pour guider ses actes.

L'infirmière débutante a fait face à suffisamment de situations réelles pour noter les facteurs signifiants qui se reproduisent dans des situations identiques. Elle a besoin d'être encadrée et a besoin d'aide pour établir les priorités.

L'infirmière compétente travaille dans le même service depuis deux ou trois ans. Elle a le sentiment de maîtriser les choses et d'être capable d'y faire face.

L'infirmière performante perçoit les situations comme des touts et non en termes d'aspects. Elle apprend par l'expérience quels événements typiques risquent d'arriver et s'oriente directement sur le problème.

L'infirmière experte a une énorme expérience et comprend de manière intuitive chaque situation. Elle appréhende directement le problème sans se perdre dans un large éventail de solutions (voir Benner, 1995, p.23-33) .

À notre avis, cette classification, même si peu d'auteurs en font état, confirme que la compétence s'accroît avec le temps et l'expérience. Si nous comparons les définitions 
du mot «compétence» proposées par les milieux de l'éducation et celles utilisées dans le domaine des soins infirmiers, nous remarquons qu'il y a certaines ressemblances. En effet, nous avons remarqué que les définitions de Barbes en éducation (1990) et celle de l'OIIQ en soins infirmiers (1980) se rejoignent lorsqu'elles font référence à des normes d'exercice, à des connaissances scientifiques ainsi qu'au jugement et aux habiletés.

Dans un deuxième temps, nous aimerions souligner que les organismes qui ont tenu les discours qui constituent notre corpus, ont énuméré les nombreuses compétences requises pour exercer le métier d'infirmière, sans avoir préalablement précisé leur conception de la notion de compétence. En effet, si tous ces organismes font référence à des exigences et à des qualités, aucun d'entre eux ne fait référence à la notion de "compétences». Toutefois, comme nous l'avons rapporté auparavant, la Corporation professionnelle des infirmières et infirmiers du Québec, en 1973, a défini l'acte infirmier à l'article 36 de la Loi des infirmières et infirmiers:

Constitue l'exercice de la profession d'infirmière ou d'infirmier tout acte qui a pour objet d'identifier les besoins de santé des personnes, de contribuer aux méthodes de diagnostic, de prodiguer et de contrôler les soins infirmiers que requièrent la promotion de la santé, la prévention de la maladie, le traitement et la réadaptation, ainsi que le fait de prodiguer des soins selon une ordonnance médicale (p.12).

À notre avis, c'est à partir de cette définition que nous pouvons dégager la compétence infirmière. L'OIIQ précise dans le document Extraits de l'évaluation de la compétence professionnelle de l'infirmière et de l'infirmier au Québec (1984) que les normes et les critères de compétence doivent être considérés comme un point de départ à la description de la compétence professionnelle (1984). Donc, si le terme «compétence» est relativement nouveau au Québec dans l'élaboration des programmes ministériels d'enseignement, il en est autrement pour l'Ordre professionnel des infirmières et infirmiers du Québec qui l'utilise depuis déjà de nombreuses années pour l'évaluation de ses membres. 
Ces précisions nous ramènent au nouveau programme Soins infirmiers 180.A0, élaboré selon l'approche par compétences, par le MEQ en 1999. Ce nouveau programme, en plus d'être encadré par la Loi sur les infirmières et les infirmiers, rend compte des nouvelles réalités en matière de santé et de soins (MEQ, 1999). Il s'inspire des concepts intégrateurs, des croyances et des postulats retenus par l'OIIQ comme assises de l'exercice à la profession (MEQ, 1999). Ces croyances sous-tendent la façon de voir la personne (famille, groupe ou collectivité), la santé, l'environnement et le soin (Perspectives de l'exercice de la profession d'infirmière, OIIQ, 1996). Le document Perspectives de l'exercice de la profession d'infirmière, publié en 1996, remplace les Normes et critères de compétence pour les infirmières et les infirmiers; Extraits de l'évaluation de la compétence professionnelle de l'infirmière et de l'infirmier au Québec. Comme le souligne l'OIIQ, ce document tient compte des nouvelles orientations du système de santé. Ces nouvelles orientations ainsi que la réforme de l'éducation sont également à la base du nouveau programme d'études collégiales en soins infirmiers. La prévision de nouvelles pratiques de soins infirmiers, comme le démontre l'analyse du contexte professionnel, exige de nombreuses qualités professionnelles chez les infirmières. En plus d'être des dispensatrices de soins, les infirmières devront être responsables, autonomes et polyvalentes et être en mesure de fonctionner dans une grande variété de rôles. En effet, des tâches variées sont à prévoir pour les infirmières: supervision, coordination d'activités, enseignement, animation de l'équipe de travail et pivot de l'équipe multidisciplinaire. La formation, selon l'approche par compétences, devra permettre l'atteinte de ces compétences en tenant compte de la modification du cadre social dans lequel linfirmière exercera son rôle professionnel.

Pour le MEQ (1999), «la maîtrise de la fonction de travail, telle que définie pour les débutantes et les débutants, est assurée par un ensemble de compétences particulières permettant à l'infirmière et à l'infirmier de fournir des soins à différentes clientèles du ré- 
seau de la santé»(p.4). Ces compétences, le MEQ (1999) en a tenu compte lors de l'élaboration de son Projet de programme en soins infirmiers selon l'approche par compétences.

Le nouveau programme collégial en soins infirmiers, selon l'approche par compétences, fait suite à l'analyse des situations de travail des infirmières effectuée par le MEQ en 1996. Il faut rappeler que cette analyse constitue l'amorce d'un long processus d'élaboration d'un programme de formation selon l'approche par compétences. Le but de cette analyse étant d'identifier les éléments de la situation de travail pour lesquels une formation est requise, elle implique un relevé des tâches, des opérations et des sous-opérations significatives en rapport avec une fonction de travail (Touzin, 1993). Concernant les soins infirmiers, cette étape a eu lieu précisément en octobre 1996, tel que nous l'avons rapporté précédemment. Puis, de cette analyse, des habiletés et des comportements jugés essentiels à l'exercice de la profession ont été dégagés et des suggestions concernant la formation ont été collectées (MEQ, 1996). Par la suite, la définition des buts et des compétences à développer a été présentée pour validation auprès des spécialistes de la profession et des conseillers techniques (Touzin, 1993). Suite à cette étape, des énoncés de compétences professionnelles en soins infirmiers, appelés compétences spécifiques, ont été énumérés dans le Projet de programme en soins infirmiers 180.A0. «Les compétences spécifiques sont liées à la maîtrise du métier et se réfèrent plus immédiatement à l'exercice des tâches, la réalisation des activités et au fonctionnement dans le cadre du travail» (Tremblay: cité dans Lavoie et Painchaud, 1993). Suivra la phase d'élaboration des plans cadres qui serviront à détailler les compétences avant de procéder à la mise en forme du programme lui-même. Les Cégeps de la province, avec la collaboration des enseignants de la formation fondamentale et technique, en sont actuellement aux dernières étapes de 
l'approche par compétences, c'est-à-dire l'élaboration des plans-cadres et des plans de cours.

L'approche par compétences est beaucoup plus complexe que ce que nous venons de décrire dans ce texte; toutefois, nous considérons que ce minimum d'informations permet de situer la formation en soins infirmiers dans un contexte de compétences. Pour l'enseignement des soins infirmiers, 22 compétences spécifiques sont visées:

1. Analyser la fonction de travail.

2. Développer une vision intégrée du corps humain et de son fonctionnement.

3. Composer avec les réactions et les comportements d'une personne.

4. Se référer à une conception de la discipline infirmière pour définir sa pratique professionnelle.

5. Utiliser des méthodes d'évaluation et des méthodes de soins.

6. Établir une communication aidante avec la personne et ses proches.

7. Composer avec des réalités sociales et culturelles liées à la santé.

8. Relier des désordres immunologiques et des infections aux mécanismes physiologiques et métaboliques.

9. Interpréter une situation clinique en se référant aux pathologies et aux problèmes relevant du domaine infirmier.

10. Établir des liens entre la pharmacothérapie et une situation clinique.

11. Enseigner à la personne et à ses proches.

12. Assister la personne dans le maintien et l'amélioration de sa santé.

13. S'adapter à différentes situations de travail.

14. Établir des relations de collaboration avec les intervenantes et les intervenants.

15. Intervenir auprès d'adultes et de personnes âgées hospitalisés requérant des soins infirmiers de médecine et de chirurgie.

16. Concevoir son rôle en s'appuyant sur l'éthique et sur les valeurs de la profession.

17. Appliquer des mesures d'urgence.

18. Intervenir auprès d'une clientèle requérant des soins infirmiers en périnatalité.

19. Intervenir auprès d'enfants ainsi que d'adolescentes et d'adolescents requérant des soins infirmiers.

20. Intervenir auprès des personnes recevant des soins infirmiers en médecine et en chirurgie dans des services ambulatoires.

21. Intervenir auprès des personnes requérant des soins infirmiers en santé mentale. 
22. Intervenir auprès d'adultes et de personnes âgées en perte d'autonomie requérant des soins infirmiers en établissement.

Sont aussi précisés dans le programme, le contexte de réalisation, les éléments de compétence et les critères de performance en lien avec chaque énoncé de compétence. La matrice compte aussi des compétences dites «générales» portant sur la formation fondamentale qui complètent le programme d'enseignement collégial en soins infirmiers.

L'analyse des discours officiels, dont les résultats ont été présentés dans le chapi tre antérieur, nous a permis de constater que l'ensemble des organismes sont d'accord pour dire que la pratique infirmière suppose l'acquisition de connaissances dans des domaines aussi variés que la psychologie, la sociologie, la pharmacologie, la biologie, l'anatomie, l'éthique, les rapports interethniques et interculturels, etc. Certaines compétences $(2,3,4,7,8,9$ et 10$)$ du nouveau programme semblent répondre à cette demande.

2. Développer une vision intégrée du corps humain et de son fonctionnement.

3. Composer avec les réactions et les comportements d'une personne. Éléments de la compétence:

3. Interpréter les réactions et les comportements de la personne.

6. Réagir à des situations à risque.

4. Se référer à une conception de la discipline infirmière pour définir sa pratique professionnelle.

7. Composer avec des réalités sociales et culturelles liées à la santé. Éléments de la compétence:

1. Considérer le contexte social dans lequel s'inscrit sa pratique professionnelle.

2. Tenir compte du contexte familial de la personne.

3. Tenir compte des caractéristiques socioculturelles de la personne.

4. Tenir compte du ou des problèmes sociaux de la personne.

8. Relier des désordres immunologiques et des infections aux mécanismes physiologiques et métaboliques. 
Éléments de la compétence:

1. Préciser l'action d'un agent agresseur sur l'organisme humain.

3. Décrire le désordre infectieux ou immunologique.

5. Examiner les mesures de prévention ou de contrôle de l'infection.

9. Interpréter une situation clinique en se référant aux pathologies et aux problèmes infirmiers.

Éléments de la compétence:

1. Décrire le problème de santé.

2. Relier les résultats des examens et le problème de santé diagnostiqué.

4. Cerner les problèmes infirmiers en lien avec la situation de santé.

10. Établir des liens entre la pharmacothérapie et une situation clinique. Éléments de la compétence:

2. Lier les manifestations cliniques et le médicament prescrit.

3. Anticiper les effets du médicament.

4. Préciser les conditions d'administration du médicament.

Quant aux sens des responsabilités et à l'autonomie professionnelle, les compétences 4 et 17 semblent rejoindre les demandes actuelles:

4. Se référer à une conception de la discipline infirmière pour définir sa pratique professionnelle.

Éléments de la compétence:

1. Appuyer son action sur les assises de l'exercice professionnel.

2. $\quad$ Se baser sur un modèle conceptuel dans l'exercice de ses fonctions.

17. Concevoir son rôle en s'appuyant sur l'éthique et sur les valeurs de la profession.

Éléments de la compétence:

1. Adhérer aux valeurs de la profession.

2. Se responsabiliser par rapport à ses actes et à ses décisions.

3. Saisir la dimension sociale de son rôle.

4. Considérer différentes situations d'un point de vue éthique.

5. S'engager dans le maintien et l'amélioration de la qualité des soins infirmiers.

Le nouveau programme de Soins infirmiers 180.A0 élaboré selon l'approche par compétences nous semble répondre «en partie» aux demandes actuelles en soins de san 
té. En effet, dans son nouveau programme, le ministère de l'Éducation a identifié les compétences professionnelles de l'infirmière et, par la suite, élaboré un programme d'études qui colle davantage à la réalité du travail. Cependant, nous croyons que la nouvelle formation collégiale permettra de développer une «certaine» autonomie, le sens des responsabilités et les habiletés en communication; d'établir une bonne communication avec les autres intervenants; et de s'adapter à différents contextes d'intervention dans les lieux ciblés pour la pratique. Si le nouveau programme collégial en soins infirmiers élaboré selon l'approche par compétences est en mesure de répondre partiellement aux nouvelles demandes sociales (autonomie, sens de responsabilité, haut niveau de connaissances, etc.), c'est par la poursuite d'études universitaires que les infirmières pourront, éventuellement, franchir un autre pas vers la professionnalisation. Le continuum de formation sur cinq ans, proposé par le MEQ à l'hiver 2001 en facilitant l'accès aux études universitaires pour les futures infirmières, permettra l'obtention d'un diplôme de baccalauréat au terme de deux ans de scolarité et contribuera ainsi à la formation de professionnelles de haut niveau. Toutefois, le maintien de deux programmes de formation de base, l'un de niveau collégial et l'autre de niveau universitaire, continuera de diviser la profession et demeurera un obstacle majeur à l'avancement de la profession.

Pour en revenir au nouveau programme collégial en soins infirmiers, nous croyons que certains éléments pourraient influencer l'implantation et la réussite de ce nouveau programme: la durée totale de la formation, les préalables à l'inscription ainsi que le manque de préparation des enseignantes à cette nouvelle approche. Ces trois éléments, à notre avis, pourraient susciter de la controverse lors de l'implantation du nouveau programme. Sans développer en détail chacun des éléments, nous aimerions avancer quelques points qui nous apparaissent problématiques. 
Dans un premier temps, nous aimerions rappeler que malgré l'ajout de nouveaux contenus, entre autres en pharmacologie, aucune heure supplémentaire n'a été ajoutée à la durée actuelle de la formation collégiale en soins infirmiers. En effet, les programmes Soins infirmiers 180.01 et $180 . A 0$ totalisent chacun 2115 heures contact. Dans les faits, cela veut dire que pas une heure supplémentaire n'a été ajoutée à la durée de la formation actuelle. Comment favoriser l'atteinte de toutes les compétences énumérées sans augmenter la durée totale de la formation? Le ministère souhaite donc augmenter les connaissances des infirmières de formation collégiale mais en gardant le même nombre d'heures d'enseignement. Rappelons que l'ajout d'une quatrième année de formation avait été recommandé par le comité Rodger et que cette recommandation n'a jamais été suivie. Est-il réaliste de développer autant de compétences dans une formation de niveau collégial? Il nous semble utopique de croire qu'on peut former de meilleures infirmières en ajoutant des nouveaux contenus sans augmenter la durée de la formation et sans préparer le corps enseignant aux nouvelles exigences.

De plus, nous pensons qu'il serait souhaitable d'exiger certains préalables à la formation collégiale en soins infirmiers dont la réussite d'un cours de biologie et d'un cours de mathématiques avancées. Cela se justifie car l'enseignement des soins infirmiers réfère continuellement à des notions de biologie pour l'étude des pathologies ainsi qu'à celui de mathématiques, base dans le calcul des doses de médicaments. Il serait donc souhaitable que les étudiantes inscrites dans un programme de soins infirmiers puissent fixer leurs nouvelles connaissances sur des acquis antérieurs. Or, il n'y a pas de préalable en biologie pour l'étudiant qui s'inscrit en soins infirmiers, pas plus que l'exigence d'avoir réussi le cours de mathématiques de niveau supérieur au secondaire. Les conditions particulières d'admission au programme collégial en soins infirmiers sont d'avoir réussi les cours suivants de niveau secondaire: Sciences physiques 436 et Chimie 534. 
Enfin, nous croyons que le changement de philosophie de l'enseignement selon l'approche par compétences a, comme le rapporte Perrenoud (1995, p.6), des incidences sur le métier d'enseignant: «[...] l'approche par compétences amène le personnel enseignant à travailler sur des situations-problèmes, dans le cadre d'une pédagogie du projet, en même temps qu'elle demande aux élèves d'être actifs et engagés dans leurs apprentissages».

Puisqu'il s'agit de changements importants pour les enseignantes, nous croyons qu'elles devraient recevoir la formation appropriée avant que le nouveau programme soit implanté dans les Cégeps de la province. En effet, cette nouvelle approche implique plusieurs changements pour le personnel enseignant, autant au niveau de la planification de l'enseignement qu'au niveau de l'évaluation des apprentissages. Concernant l'enseignement, Perrenoud (1995) souligne que dorénavant, le métier d'enseignant ne consistera plus à enseigner mais à faire apprendre, en créant des situations favorables aux apprentissages. Il semble, selon cet auteur, que la situation-problème place l'apprenant devant une série de décisions à prendre pour atteindre un objectif, contrairement à une situation didactique quelconque. Il s'agit donc, pour viser le développement de compétences, de "se creuser la tête» pour créer des situations-problèmes à la fois mobilisatrices et orientées vers des apprentissages spécifiques. La création continue de nouvelles situations-problèmes, impossibles à fabriquer par un seul enseignant, implique toujours, selon le même auteur, que de nouveaux outils didactiques doivent être disponibles rapidement afin de permettre aux enseignants de travailler dans le sens de l'approche par compétences.

Au sujet de l'évaluation, l'implantation de la réforme suppose de nouvelles méthodes d'évaluation. En effet, l'évaluation formative prendra une grande importance com- 
me le souligne Lasnier (2000). L'évaluation formative, telle que définie par Scallon, consiste en:

Un processus d'évaluation continue ayant pour objectif d'assurer la progression des individus engagés dans une démarche d'apprentissage ou de formation, selon deux voies possibles: soit par des modifications de la situation ou du contexte pédagogique, soit en offrant à chaque individu l'aide dont il a besoin pour progresser, et ce, dans chacun des cas, pour apporter, s'il y a lieu, des améliorations ou des correctifs appropriés. La «décision action», c'est-à-dire la régulation, a pour objet soit la situation d'apprentissage, soit l'individu lui-même (2000: cité dans Lasnier, 2000, p.220).

Ce processus d'évaluation n'est pas inconnu aux enseignantes des collèges en soins infirmiers. En effet, en soins infirmiers, depuis plus de dix ans, l'évaluation formative est une pratique courante autant en laboratoire collège qu'en laboratoire clinique (DGEC, 1987). Cependant, ce qui est nouveau, c'est que l'article 35 du nouveau Règlement sur le régime des études collégiales prévoit, pour tous les programmes de formation, l'établissement d'une épreuve synthèse, au terme de la formation pré-universitaire et technique (Matteau, 1994-1995). Cette évaluation, au terme du programme, vise à attester de l'intégration des apprentissages réalisés dans l'ensemble du programme de formation. Elle peut prendre différentes formes selon le programme d'études et doit être élaborée, administrée et corrigée par les enseignants de chaque programme (Matteau, 1994-1995). Pour les étudiantes infirmières, il s'agit d'une nouvelle évaluation, de fin de programme, qui s'ajoute à celle tenue par l'OIIQ lors de l'administration de l'examen professionnel donnant accès au droit de pratique.

Pour revenir aux étapes qui ont permis l'avancement du dossier de la formation, c'est en décembre 2000 que le comité provincial sur la formation infirmière remettait son rapport à la Sous-ministre. Le Comité suggère un programme de formation DEC-BAC intégré, c'est-à-dire trois ans d'études collégiales avec accès au droit de pratique suivi de 
deux ans d'université pour l'obtention d'un baccalauréat en sciences infirmières. Les cibles retenues pour l'enseignement au niveau collégial sont la personne et ses proches alors que celles de l'enseignement universitaire sont principalement axées sur la personne, la famille, le groupe et la communauté. La formation universitaire permet à l'infirmière d'élaborer, d'offrir et d'évaluer des programmes de soins, d'enseignement et d'éducation à la santé de niveau universitaire, alors que l'infirmière de formation collégiale prodigue un enseignement à partir d'un plan d'enseignement qui a été élaboré par une infirmière bachelière. De plus, l'infirmière formée à l'université acquiert le bagage de connaissances, d'habiletés intellectuelles et professionnelles, de valeurs et d'attitudes nécessaires à une pratique autonome et sécuritaire. Le volet «soins communautaires» demeure donc, essentiellement, de niveau universitaire. Malgré la persistance des deux ordres de formation de niveau supérieur en soins infirmiers, le statu quo, il semble y avoir une volonté ferme d'articuler les deux programmes afin de former des infirmières qui seront mieux formées pour répondre aux demandes de la santé. D'ailleurs, c'est dans cette optique, que le 26 janvier 2001 , le MEQ signifiait aux enseignantes des collèges et des universités l'obligation de s'entendre sur l'élaboration d'un continuum de formation. Nous pouvons constater qu'il y a aujourd'hui, de la part du MEQ, une ouverture au changement qui, il y a à peine une décennie, était impensable. Il aura fallu deux réformes, celle de la santé et celle de l'éducation, pour faire avancer le débat sur la formation infirmière.

Au terme de nos analyses, il nous apparaît que la formation collégiale en soins infirmiers, même si elle semble répondre aux besoins de certains établissements de santé du secteur public, est insuffisante pour répondre aux demandes actuelles en soins de santé qui sont davantage axés sur le travail dans la communauté. Toutefois, les compétences acquises au cours de la formation collégiale permettront, à la future infirmière de niveau collégial, d'acquérir plus d'autonomie, d'augmenter légèrement ses connaissances et son 
sens de l'initiative, mais elle demeurera une formation de base. Pour accéder à des fonctions supérieures, l'infirmière devra poursuivre sa formation à l'université. De plus, pour répondre aux nouvelles demandes sociales spécifiques à la santé communautaire, à l'élaboration de plans d'enseignement, à la gestion, ainsi qu'aux nombreuses autres exigences, l'infirmière devra obligatoirement avoir une formation universitaire en sciences infirmières.

Nous sommes convaincue que le continuum de formation sur cinq ans sera une formule gagnante pour la profession infirmière. 


\section{CONCLUSION}

Notre but était d'examiner, par une analyse de contenu effectuée à partir de textes publiés par des organismes qui s'intéressent à la formation infirmière, si la formation collégiale en soins infirmiers pouvait répondre aux demandes actuelles en soins de santé.

Pour trouver des éléments de réponse à notre questionnement, nous avons traité trois thèmes qui nous semblaient en lien direct avec les soins infirmiers. Il nous a fallu d'abord connaître les nouvelles demandes sociales en soins infirmiers pour voir, dans un deuxième temps, dans quelle mesure elles influençaient l'aspect professionnel des soins infirmiers. Enfin, nous avons voulu savoir si la formation collégiale en soins infirmiers permettait de répondre aux nouvelles demandes sociales.

C'est par une analyse de contenu thématique, effectuée sur un corpus de textes officiels, que nous avons pu recueillir des données significatives nous permettant de faire évoluer la réflexion et être en mesure de répondre à nos questions de recherche. Cependant, c'est à cette étape de la recherche que nous avons connu quelques difficultés. En effet, comme nous n'étions pas familière avec ce type d'instrument, il nous fallait être très prudente pour garder le sens des discours dans la recherche d'une réponse à notre question qui était: la formation collégiale en soins infirmiers peut-elle répondre aux demandes sociales actuelles en soins de santé ? C'est en divisant les textes en trois grands thèmes de base: le contexte social, le contexte professionnel et le contexte de formation que nous 
avons procédé à l'analyse de contenu et ressorti les points marquants des discours. Ceci nous a permis de constater que tous les organismes étaient unanimes à dire que les changements récents dans le réseau de la santé auront des conséquences majeures sur la pratique infirmière et demanderont l'acquisition de nouvelles exigences pour ce corps professionnel. Cependant, au niveau de la formation de base en soins infirmiers, nous avons noté des divergences significatives de la part de ces mêmes organismes.

Si la position de plusieurs organismes nous est apparue claire, il en fut autrement pour le MEQ qui ne s'est jamais positionné franchement dans son discours, sur la question de la formation de base en soins infirmiers avant l'implantation du nouveau programme en soins infirmiers selon l'approche par compétences. Nous avons procédé à une brève analyse de ce nouveau programme afin de savoir dans quelle mesure il pourrait permettre de répondre aux nouvelles demandes sociales. Nous pensons que l'implantation du nouveau programme collégial en soins infirmiers, selon l'approche par compétences, prévue pour l'automne 2001 dans tous les collèges offrant la formation en soins infirmiers, est précipitée. À notre avis, un délai supplémentaire aurait permis aux enseignants/es de se familiariser davantage le contenu du nouveau programme ainsi que l'approche par compétences afin de se l'approprier avant d'assumer les tâches liées à l'enseignement. La rapidité d'implantation pourrait, à notre avis, avoir des répercussions majeures sur la qualité de la formation que recevront plusieurs milliers d'étudiants en soins infirmiers dans les prochaines années. Il aurait été préférable, à notre avis, de cibler quelques collèges afin de réaliser des expériences pilote avant d'implanter massivement le nouveau programme dans les 42 collèges. Cette expérimentation permettrait d'évaluer dans quelle mesure il saura répondre aux demandes sociales actuelles. C'est en 2004 qu'une première cohorte accédera au marché du travail. Une autre étude pourrait être entreprise à ce mo- 
ment afin d'analyser si la formation collégiale, selon l'approche par compétences, a permis de répondre aux demandes sociales en soins de santé.

Ainsi, malgré le souhait de certaines instances de voir la formation de base infirmière de niveau universitaire, le MEQ a statué pour le maintien de la formation de base en soins infirmiers de niveau collégial et de la formation initiale universitaire en sciences infirmières. De cette manière, le MEQ prescrit le statu quo. Cependant, la formation universitaire devient une option très intéressante pour les finissantes du programme collégial puisqu'un continuum de formation sur cinq ans permettra l'obtention du baccalauréat en sciences infirmières. Ainsi, dans le contexte actuel de pénurie de main-d'œuvre en soins infirmiers, le maintien de la formation collégiale avec accès au droit de pratique après trois ans demeure la solution la plus avantageuse pour les ministères de l'Éducation et de la Santé et des Services sociaux. De plus, tout en permettant l'accès au marché du travail au terme de trois ans d'études collégiales, la formation universitaire raccourcie encouragera la poursuite d'études universitaires pour les infirmières finissantes des collèges.

Cette recherche qualitative nous a permis de constater, au terme de plusieurs lectures et analyses, que malheureusement la formation initiale unique en soins infirmiers ne fait pas l'unanimité et que, par conséquent, elle demeurera un sujet d'actualité très stimulant pour les chercheurs des milieux de l'éducation et de la santé.

Nous n'avons pas la prétention d'avoir fait le tour du sujet puisque nous n'avons pas analysé l'ensemble des documents publiés sur le sujet, pas plus qqu'assister aux rencontres de discussions organisées par des intervenants intéressés à cette problématique. Toutefois, l'analyse de contenu nous a permis de constater, qu'encore aujourd'hui, le ni- 
veau de formation des infirmières demeure un sujet très controversé malgré l'élargissement du rôle professionnel découlant des transformations du réseau de la santé. En effet, l'analyse des documents officiels que nous avons réalisée a permis de mettre en évidence les compétences requises tandis que l'analyse du nouveau programme collégial en soins infirmiers, quoique très restreinte, nous a permis de constater que les compétences du programme sont insuffisantes pour former des infirmières pouvant travailler dans l'ensemble du réseau de la santé. Les infirmières formées au niveau collégial devront donc poursuivre leur formation à l'université si elles désirent élargir leur champ de pratique. Cependant, la formation initiale universitaire en sciences infirmières permet-elle de former des infirmières en mesure de répondre aux demandes actuelles en soins de santé? Une autre recherche, appliquée à la formation initiale en soins infirmiers de niveau universitaire, pourrait permettre de faire cheminer davantage les dossiers de la formation infirmière et de la professionnalisation. De plus, il serait intéressant d'entreprendre, dans quelques années, une étude permettant de vérifier si le continuum de formation sur cinq a permis de mieux répondre aux demandes sociales. 


\section{BIBLIOGRAPHIE}

ADAM, E. (1991). Être infirmière; un modèle conceptuel. Montréal:Éditions Études Vivantes, p.49-56.

ANADÓN, M. (1999). «L'enseignement en voie de professionnalisation», dans C., Gohier, N. Bednarz, L. Gaudreau, R. Pallascio et G. Parent (dir.), Regards sur l'enseignant professionnel. Québec : Presses de l'Université du Québec, p.1-20.

ASSOCIATION DES DIRECTEURS ET RESPONSABLES DES SOINS INFIRMIERS DU QUÉBEC (1993). L'offre future d'infirmières autorisées au Canada. Document de travail, $21 \mathrm{p}$.

ASSOCIATION DES INFIRMIÈRES ET INFIRMIERS DU CANADA (1997). L'évolution des soins infirmiers au Québec: pour une dynamique d'amélioration continue. Québec: La réflexion de l'ADRSIQ: document non publié, $27 \mathrm{p}$.

BARDIN, L. (1977). L'analyse de contenu. Paris: Presses Universitaires de France, 288 p.

BEAULIEU, C. (1997). «Le virage c'est elles!», L'Actualité, juin, p.15-35.

BENNER, P. (1995). De novice à expert. Excellence en soins infirmiers. Québec: ERPI, $251 \mathrm{p}$.

BIBLIOTHĖQUE NATIONALE DU CANADA (2000). Guide d'admission à l'université. Tout ce qu'il faut savoir et faire pour réussir votre entrée universitaire. Québec: Éditions Septembre, Collection «Choisir Enr.», p.14-15.

BLONDEAU, D. (1986). De l'éthique à la bioéthique; repères en soins infirmiers. Québec: Gaétan Morin, p.31-75.

BLONDEAU, D. (1993). "Les valeurs de la profession infirmière d'hier à aujourd'hui», dans O. Goulet (dir.), La profession infirmière. Valeurs, enjeux, perspectives. Québec: Gaétan Morin, p.7-22.

BLONDEAU, D. (1999). Éthique et soins infirmiers. Montréal: Les Presses de l'Université de Montréal, $336 \mathrm{p}$. 
BLOUN, H. (1994). «La réintégration des services sociaux hospitaliers: vers de nouvelles initiatives», Intervention, $\mathrm{n}^{\circ} 98$, juin, p.26-31.

BOLDUC, D. (1997). Le Suivi Systématique de clientèles. Chicoutimi: Formation donnée au Cégep de Chicoutimi, 25 avril (texte non publié).

CARPENITO, L.J. (1997). Préface. Plans de soins et dossier infirmier. Diagnostics infirmiers et problèmes traités en collaboration. Paris: Éditions De Boeck Université, p.IX.

COHEN, Y. (2000). Profession infirmière. Une histoire des soins dans les hôpitaux du Québec. Montréal: Les Presses de l'Université de Montréal, $321 \mathrm{p}$.

DALLAIRE, C. (1999). «Les grandes fonctions de la pratique infirmière», dans O. Goulet et C. Dallaire (dir), Soins infirmiers et société. Québec: Gaétan Morin, p.33-51.

DESJARDINS, E., GIROUX, S. et FLANAGAN, E.C. (1970). Histoire de la profession infirmière au Québec. Montréal: Éditions du Richelieu Ltée, 270 p.

DIRECTION GÉNÉRALE DE L'ENSEIGNEMENT COLLÉGLAL (1987). Document d'accompagnement du programme Soins infirmiers 180.01. Québec: MEQ, Service des programmes, $213 \mathrm{p}$.

DOENGES, M.E. et al. (1996). Guide pratique. Diagnostics infirmiers et interventions. Montréal: ERPI, p.8-9.

DOYE, M. (1997). La pratique de première ligne en soins infirmiers. Chicoutimi: Formation donnée au Cégep de Chicoutimi, 5 et 6 juin (texte non publié).

DUNNIGAN, L. (1996). Info-Santé CLSC. Service téléphonique accessibilité continue 24/7. Étude préliminaire et premières propositions en vue de l'évaluation des résultats et de l'impact du service. Québec: Direction de la Recherche et de l'Évaluation, Direction générale de la planification et de l'Évaluation, Ministère de la Santé et des Services sociaux, $66 \mathrm{p}$.

FÉDÉRATION DES INFIRMIĖRES ET INFIRMIERS DU QUÉBEC (1995). Mémoire déposé dans le cadre de la Commission des États généraux sur l'éducation. Québec: FIIQ, $50 \mathrm{p}$.

FORTIER-HAVELKA, C. (1986). Profil de l'infirmière en début de carrière et sa performance au travail. Rapport de recherche subventionnée par les fonds FCAR, Programme A.C.S.A.I.R., 24 p. 
FOURNIER, L. (1999). «La pratique infirmière contemporaine dans le système de santé québécois», dans O. Goulet et C. Dallaire (dir.), Soins infirmiers et société. Québec: Gaétan Morin, p.7-29.

GAUTHIER, C. et TARDIF, M. (2000). «Mais moi, comment j'ai appris à enseigner ? Et comment puis-je savoir que je suis compétent?», Vie pédagogique, $\mathrm{n}^{\circ} 117$, novdéc., p.15-19.

GERMAIN, R. (1985). Le mouvement infirmier au Québec, 50 ans d'histoire. Québec: Bellarmin, $565 \mathrm{p}$.

GOULET, J.P. (1994). «Pour en finir avec "la réforme"», Pédagogie collégiale, vol. 8, n² 2.

GOULET, O. et DALLAIRE C. (1999). Soins infirmiers et société. Québec: Gaétan Morin, $273 \mathrm{p}$.

GOULET, O. (1993). «Les infirmières et la formation universitaire», dans O. Goulet (dir.), La profession infirmière. Valeurs, enjeux, perspectives. Québec: Gaëtan Morin, p.179-206.

GOUVERNEMENT DU QUÉBEC (1996). Virage ambulatoire: notes exploratoires. Québec: Gouvernement du Québec, Conseil du statut de la femme, $27 \mathrm{p}$.

GOUVERNEMENT DU QUÉBEC (1996). Santé Infirmière diplômée et infirmier diplômé de l'université. Rapport d'analyse de situation de travail. Québec: Ministère de l'Éducation. Direction des programmes. Direction générale de la formation professionnelle et technique. Hélène L'Heureux. Version non validée, 13 novembre 1996.

GOUVERNEMENT DU QUÉBEC (1996). Santé Infirmière diplômée et infirmier diplômé du collégial. Rapport d'analyse de situation de travail. Québec: Ministère de l'Éducation. Direction des programmes. Direction générale de la formation professionnelle et technique. Hélène L'Heureux. Version non validée, 11 novembre 1996.

GOUVERNEMENT DU QUÉBEC (1996). Service téléphonique accessibilité continue 24/7. Étude préliminaire et premières propositions en vue de l'évaluation des résultats et de l'impact du service. Québec: Ministère de la Santé et des Services sociaux, Direction de la Recherche et de l'Évaluation, Direction générale de la Planification et de l'Évaluation. Info-Santé CLSC, Lise Dunnigan, Québec: 92 p.

GOUVERNEMENT DU QUÉBEC (1997). Bulletin de santé. Québec: Ministère de la Santé et des Services sociaux, Québec, $8 \mathrm{p}$.

GOUVERNEMENT DU QUÉBEC (2000). Projet de programme soins infirmiers 180.A0. Québec: Direction générale de la formation professionnelle et technique, $123 \mathrm{p}$. 
HENDERSON, V. (1994). La nature des soins infirmiers. Québec: Éditions du Renouveau pédagogique, $235 \mathrm{p}$.

KÉROUAC, S., PÉPIN, J., DUCHARME, F., DUQUETTE, A. et MAJOR, F. (1994). La pensée infirmière. Québec: Éditions Études vivantes, ch. 5, p.97-114.

LAFERRIÈRE, S. (1994). «L'approche par compétences», Pratiques Pédagogiques, mars p.3-5.

LALANCETTE, D. (1993). «L'ordre professionnel», dans O. Goulet (dir.), La profession infirmière. Valeurs, enjeux, perspectives. Québec: Gaëtan Morin, p.93-112.

LAMBERT, C. (1993). «La formation infirmière dans les cégeps», dans O. Goulet (dir.), La profession infirmière. Valeurs, enjeux, perspectives. Québec: Gaëtan Morin, p.149-177.

LAROUCHE, R. (1997). La sociologie des professions; perception critique de certains concepts ou courants d'idées. Québec: Bibliothèque Nationale du Québec, $170 \mathrm{p}$.

LASNIER, F. (2000). Réussir la formation par compétences. Montréal: Éditions Guérin, $485 \mathrm{p}$.

LAVOIE, C. et PAINCHAUD, J. (1993). «La compétence: une définition», Pédagogie collégiale, vol. $6, \mathrm{n}^{\circ} 3$, p.17.

LEFEBVRE, H. et BRULÉ, M. (1997). «L'examen physique: où en sommes-nous?», L'infirmière du Québec, juillet/août, p.42.

LEGENDRE, R. (1993). Dictionnaire actuel de l'éducation, $2^{\mathrm{e}}$ édition. Montréal: Éditions Guérin.

MATTEAU, P. (1994-1995). «L'épreuve synthèse de programme: on s'y prépare ...», Reflets, vol. $5, \mathrm{n}^{\circ} 5, \mathrm{p} .2-3$.

MAYER, R. et OUELLET, F. (1991). Méthodologie de recherche pour les intervenants sociaux. Québec: Gaëtan Morin Éditeur, 537 p.

ORDRE DES INFIRMIÈRES ET INFIRMIERS DU QUÉBEC (1980). L'évaluation de la compétence professionnelle de l'infirmière et de l'infirmier au Québec. Tome I. Méthode d'évaluation de la compétence professionnelle de l'infirmiere et de l'infirmier, p.5.

ORDRE DES INFIRMIĖRES ET INFIRMIERS DU QUÉBEC (1982). La formation professionnelle initiale de l'infirmière au niveau universitaire en l'an 2000..., Synthèse du rapport préliminaire, $16 \mathrm{p}$. 
ORDRE DES INFIRMIĖRES ET INFIRMIERS DU QUÉBEC (1987). L'infirmière et sa corporation professionnelle, $40 \mathrm{p}$.

ORDRE DES INFIRMIĖRES ET INFIRMIERS DU QUÉBEC (1989). Hypertension; l'urgence des choix, $150 \mathrm{p}$.

ORDRE DES INFIRMIÈRES ET INFIRMIERS DU QUÉBEC (1996). Perspectives de l'exercice de la profession d'infirmière, $29 \mathrm{p}$.

ORDRE DES INFIRMIÈRES ET INFIRMIERS DU QUÉBEC (1996). Les effectifs de la profession infirmière: analyse prospective et enjeux. Montréal: Etude réalisée par le groupe Sécor, $70 \mathrm{p}$.

ORDRE DES INFIRMIÈRES ET INFIRMIERS DU QUÉBEC (1996). Pour soigner notre avenir, les infirmières à la croisée des chemins. Actes des États généraux de la profession d'infirmière. Centre des Congrès de Québec, 6-7 mai.

PELLAND, J. (1991). «Cap sur le progrès». Allocution d'ouverture prononcée lors de la $70^{\mathrm{e}}$ assemblée générale annuelle de l'OIIQ, Nursing Québec, vol.11, $\mathrm{n}^{\circ} 1, \mathrm{p} .5$.

PERRENOUD, P. (1995). «Des savoirs aux compétences. De quoi parle-t-on en parlant de compétences?», Pédagogie collégiale, vol. $9, \mathrm{n}^{\circ} 1, \mathrm{p} .6$.

PERRENOUd, P. (1995). «Des savoirs aux compétences. Les incidences sur le métier d'enseignant et sur le métier d'élève», Pédagogie collégiale, vol. 9, n², p.20.

PETITAT, A. (1989). Les infirmières de la vocation à la profession. Québec: Boréal, ch. 2, p.41-108.

PHANEUF, M. (1986). Soins infirmiers, la démarche scientifique. Orientation vers le diagnostic infirmier. Québec: McGraw-Hill, p.50.

PÔLET-MASSET, A.M. (1993). Développer son autonomie en soins infirmiers. Québec: Gaëtan Morin, ch. 2, p.27-34

POTTER, P. (1990). Soins infirmiers. Théorie et pratique. Québec: ERPI, ch. 1, p.3-32.

RIOPELLET, L. et LEDUC-LALONDE, L. (1982). Individualisation des soins infirmiers. Québec: McGraw-Hill, ch. 1, p.1-15.

SERVICE RÉGIONAL D'ADMISSION DU MONTRÉAL MÉTROPOLITAIN (1999). Guide pratique des études collégiales. Montréal: Service régional d'admission du Montréal Métropolitain, p.363.

ST-AMOUR, G., DESGROSEILLERS, F. ET RICHARD, M. (1987). Engagement professionnel, un processus de socialisation en soins infirmiers. Québec: Décarie, p.61-98. 
TÊTU, G. (1995). Au cœur du virage ambulatoire: les impacts dans les milieux hospitaliers "Virage ou chirage». Conférence tenue le 3 octobre à l'Hôpital de Chicoutimi (texte non publié).

TOUZÎN, G. (1993). Notes de cours: Enseigner des compétences. Sherbrooke: Programme Performa, Université de Sherbrooke.

TOUZIN, G. (1994). Élaboration d'un plan de cours dans le contexte d'un enseignement par compétences. Du plan cadre au plan de cours. Chicoutimi: Éditeurs Entreprise GT Enr., 277 p.

TOUZIN, G. (1994). «L’approche par compétences», Reflets, vol. 5, n² 2, p.4.

UNIVERSITÉ LAVAL (1997). Sciences de la santé. Les programmes de premier cycle. Québec: Bureau du registraire, p.19.

VILLEDIEU, Y. (1996). «La santé à un virage», Forces, 114, p.62-67.

WALTIER, B. (1988). Construction, évolution, révolution de la pensée infirmière. Paris: Lamarre-Poirat, 275 p.

SITES WWW CONSULTÉS:

http://www.ahq.org./apropos.mission.html

http://www.fiiq.qc.ca/unedecl.htm

http://www.oiiq.org/ordre/role.html

http://www.oiiq.org/pratique/prat-religieuse.html 